\title{
MRB7260 is essential for productive protein-RNA interactions within the RNA editing substrate binding complex during trypanosome RNA editing
}

\author{
NATALIE M. MCADAMS, ${ }^{1}$ RACHEL M. SIMPSON, ${ }^{1}$ RUNPU CHEN, ${ }^{2}$ YIJUN SUN, ${ }^{1}$ and LAURIE K. READ ${ }^{1}$ \\ ${ }^{1}$ Department of Microbiology and Immunology, University at Buffalo Jacobs School of Medicine and Biomedical Sciences, Buffalo, \\ New York 14203, USA \\ ${ }^{2}$ Department of Computer Science and Engineering, University at Buffalo, Buffalo, New York 14260, USA
}

\begin{abstract}
The trypanosome RNA editing substrate binding complex (RESC) acts as the platform for mitochondrial uridine insertion/deletion RNA editing and facilitates the protein-protein and protein-RNA interactions required for the editing process. RESC is broadly comprised of two subcomplexes: GRBC (guide RNA binding complex) and REMC (RNA editing mediator complex). Here, we characterize the function and position in RESC organization of a previously unstudied RESC protein, MRB7260. We show that MRB7260 forms numerous RESC-related complexes, including a novel, small complex with the guide RNA binding protein, GAP1, which is a canonical GRBC component, and REMC components MRB8170 and TbRGG2. RNA immunoprecipitations in MRB7260-depleted cells show that MRB7260 is critical for normal RNA trafficking between REMC and GRBC. Analysis of protein-protein interactions also reveals an important role for MRB7260 in promoting stable association of the two subcomplexes. High-throughput sequencing analysis of RPS12 mRNAs from MRB7260 replete and depleted cells demonstrates that MRB7260 is critical for gRNA exchange and early gRNA utilization, with the exception of the initiating gRNA. Together, these data demonstrate that MRB7260 is essential for productive protein-RNA interactions with RESC during RNA editing.
\end{abstract}

Keywords: Trypanosoma brucei; uridine insertion/deletion RNA editing; guide RNA; kinetoplastida; guide RNA binding complex; RNA editing substrate binding complex

\section{INTRODUCTION}

Trypanosoma brucei, which is the causative agent of human African trypanosomiasis and nagana in wildlife, is a member of the class Kinetoplastea, order Trypanosomatida (d'AvilaLevy et al. 2015). Members of this class are named for their unique mitochondrial DNA, called the kinetoplast or kDNA, which in the order Trypanosomatida is comprised of a catenated network of thousands of circular DNAs (Jensen and Englund 2012; d'Avila-Levy et al. 2015). In T. brucei, kDNA contains dozens of maxicircles that encode traditional mitochondrial genes, including components of the respiratory complexes and ribosomal RNAs. Twelve of the 18 protein-coding genes in the maxicircle require posttranscriptional RNA editing by uridine insertion and deletion to generate translatable mRNAs. In addition to maxicircles, kDNA also contains thousands of minicircles encoding small trans-acting guide RNAs (gRNAs) that direct the insertion/ deletion RNA editing of the maxicircle mRNA transcripts

Corresponding author: lread@buffalo.edu 117.

Article is online at http://www.rnajournal.org/cgi/doi/10.1261/rna.065169.
(Blum and Simpson 1990; Sturm and Simpson 1990). A few mitochondrial mRNAs are only edited in small domains that require one or a few gRNAs for complete editing, and these are referred to as minimally edited mRNAs. However, the majority of the edited mitochondrial transcripts are termed pan-edited, and these require editing throughout the entire transcript as directed by dozens of gRNAs that act sequentially from $3^{\prime}$ to $5^{\prime}$ along the mRNA to generate functional open reading frames (Maslov and Simpson 1992; Lukes et al. 2005). RNA editing is essential for survival of both insect procyclic form (PF) and mammalian bloodstream form (BF) T. brucei and is conserved across the Kinetoplastea (Schnaufer et al. 2001; Stuart et al. 2005).

The enzymatic reactions of uridine insertion/deletion RNA editing, including endonuclease cleavage, uridine insertion, uridine deletion, and RNA ligation, are catalyzed by the

\footnotetext{
(C) 2018 McAdams et al. This article is distributed exclusively by the RNA Society for the first 12 months after the full-issue publication date (see http://rnajournal.cshlp.org/site/misc/terms.xhtml). After 12 months, it is available under a Creative Commons License (Attribution-NonCommercial 4.0 International), as described at http://creativecommons.org/licenses/by$\mathrm{nc} / 4.0 /$.
} 
RECC (RNA editing core complex; a.k.a. 20S editosome) (Rusché et al. 1997; Simpson et al. 2004; Lukes et al. 2005; Stuart et al. 2005; Carnes et al. 2008). Despite containing the enzymes needed for catalysis, however, purified RECC lacks both associated RNA and processivity in in vitro RNA editing assays (Carnes et al. 2012). In addition to RECC, a second complex termed the RESC (RNA editing substrate binding complex; a.k.a., MRB1) is essential for mitochondrial RNA editing in T. brucei, and together RECC and RESC comprise the RNA editing holoenzyme (Aphasizheva and Aphasizhev 2016; Read et al. 2016). Three groups independently discovered RESC using different proteins as bait, and defined the complex as components that copurify with the GAP1/2 gRNA binding proteins (Hashimi et al. 2008; Panigrahi et al. 2008; Weng et al. 2008). Subsequent tandem affinity purifications or antibody pulldowns confirmed several common RESC components and revealed both RNA-independent and RNA-enhanced associations, and a basic organization (Weng et al. 2008; Ammerman et al. 2011, 2012, 2013; Kafková et al. 2012; Aphasizheva et al. 2014; Gazestani et al. 2016). RESC is broadly comprised of two subcomplexes: GRBC (guide RNA binding complex; a.k.a. MRB1 core) and REMC (RNA editing mediator complex; a.k.a TbRGG2 subcomplex). GRBC appears to contain seven proteins that interact in an RNA-independent manner: GAP1/2, MRB8620, MRB11870, MRB3010, MRB5390, and MRB0880 (Ammerman et al. 2012; Aphasizheva et al. 2014). With the exception of a few proteins, RESC proteins are indicated by their original "MRB" descriptors, which denote the last three to five digits of the gene identifier in TriTrypDB (http://tritrypdb.org) (Ammerman et al. 2012). REMC is currently envisioned as comprised of TbRGG2, MRB8180, MRB800, MRB1860, and the redundant paralogs MRB8170/4160. It associates with GRBC in an RNA-enhanced manner, and appears to be more heterogeneous than GRBC (Ammerman et al. 2012; Kafková et al. 2012; Aphasizheva et al. 2014; Simpson et al. 2017). MRB10130, which contains domains that resemble Armadillo (ARM) or HEAT repeats, may play a role in organizing macromolecular interactions within RESC. Taken together, the current model holds that RESC serves as the platform for RNA editing and acts in a dynamic manner to facilitate the protein-protein and protein-RNA interactions that are required for the editing process.

Complicating our understanding of the editing machinery are several observations that some RESC proteins engage in interactions outside of RESC. Specifically, GAP1 forms a complex with the REH2C RNA helicase complex apart from the GRBC protein, MRB3010, and GAP1 also interacts with the non-RESC RNA binding protein, TbRGG3 (McAdams et al. 2015; Kumar et al. 2016). Moreover, GAP1 typically sediments in a heterodispersed fashion on glycerol gradients, being present in both low- and high-molecular weight fractions unlike other GRBC proteins, such as MRB3010, that peak in only a subset of fractions (Weng et al. 2008; Hashimi et al.
2009; Huang et al. 2015). These findings indicate that GAP1 functions in RNA editing outside of the defined GRBC subcomplex. The MRB8170 component of REMC also apparently interacts with, and enhances the RNA binding capacity of, numerous non-RESC RNA binding proteins (Dixit et al. 2017).

The process of uridine insertion/deletion RNA editing can be divided into multiple steps, from the initiation of editing on an mRNA to the $3^{\prime}$ to $5^{\prime}$ progression through each gRNA-directed block. Progression of editing requires both editing through a gRNA-directed block (gRNA utilization) and the subsequent removal of the completely utilized gRNA and recruitment of the next correct gRNA (gRNA exchange). Numerous studies have begun to unravel the functions of RESC and its component subcomplexes and proteins in this process. GAP $1 / 2$ are the only proteins identified to date required for gRNA stabilization (Weng et al. 2008; Hashimi et al. 2009), while the remaining GRBC components appear to impact the initiation of RNA editing as indicated by an accumulation of pre-edited mRNA in cells depleted of these proteins (Weng et al. 2008; Hashimi et al. 2009; Ammerman et al. 2011, 2012, 2013; Aphasizheva et al. 2014; Huang et al. 2015). We recently used high-throughput sequencing analysis of partially edited transcripts in cells depleted of specific RESC components in combination with a novel bioinformatic platform, termed TREAT (trypanosome RNA editing alignment tool), to address RESC protein function (Simpson et al. 2017). We demonstrated that mRNAs from cells depleted of GAP1 display both a defect in editing initiation as well as pausing in the $3^{\prime}$ to $5^{\prime}$ progression of editing consistent with a defect in gRNA exchange (Simpson et al. 2017). However, in this case, pausing at the ends of gRNA-defined blocks is potentially due to destabilization of the entire gRNA population rather than an active role for GAP1/2 in the process of gRNA exchange. Indeed, little is known about how gRNAs are discriminated and used correctly, although one previous study reported that the non-RESC RNA helicase, REH1, selectively affects gRNA exchange in a subset of mRNAs (Li et al. 2011). With regard to REMC, we demonstrated that TbRGG2 is critical for the $3^{\prime}$ to $5^{\prime}$ progression of editing and functions as an RNA binding, annealing, and unwinding protein (Fisk et al. 2008; Ammerman et al. 2010; Foda et al. 2012). More recently, our high-throughput sequencing analysis of partially edited transcripts demonstrated that TbRGG2 and MRB8180 are involved in the utilization of gRNAs (Simpson et al. 2017). At least one function of these proteins is to promote the commonly observed nonlinear path of editing that results in junctions containing incorrectly edited sequence at the $5^{\prime}$ leading edge of editing, likely through modulation of gRNA-mRNA pairing (Simpson et al. 2017). Studies from our laboratory and others addressing the function of another REMC protein, MRB8170, indicate that this protein functions differently than TbRGG2 and MRB8180. Dixit and colleagues proposed that the binding of MRB8170 to pre-mRNA marks 
the mRNA for editing initiation and facilitates the recruitment of other RESC components to ensure efficient editing (Dixit et al. 2017). In addition, we demonstrated that MRB8170 aids in the initiation of RNA editing of certain transcripts and constrains the regions of active editing (Simpson et al. 2017).

In this study, we characterize a previously unstudied RESC protein, MRB7260 (TriTrypDB ID Tb927.9.7260; named according to convention described above). MRB7260 was originally identified in a subset of RESC pulldowns (Ammerman et al. 2012; Aphasizheva et al. 2014), but may have been overlooked due to the presence of a $\mathrm{PhyH}$ domain, suggesting that it was a glycosomal contaminant. Here, we show that MRB7260 is a mitochondrially localized protein that is essential for optimal growth and RNA editing in both insect PF and mammalian BF T. brucei. MRB7260 forms numerous RESC-related complexes, including a small complex with the guide RNA-associated protein (GAP1) and other factors. Analysis of protein-protein and protein-RNA interactions in MRB7260-depleted cells shows that this protein is critical for proper RNA trafficking between REMC and GRBC subcomplexes as well as for RESC assembly. High-throughput sequencing analysis of MRB7260-depleted cells indicates a role for MRB7260 in both the $3^{\prime}$ to $5^{\prime}$ progression of editing, and suggests that this protein functions in gRNA utilization, gRNA exchange, and gRNA discrimination during the exchange process. Together, our data demonstrate that MRB7260 is essential for the productive association of RNA and proteins within RESC during trypanosome RNA editing.

\section{RESULTS}

\section{MRB7260 is essential for $T$. brucei cellular growth and RNA editing}

MRB7260 (TriTrypDB ID Tb927.9.7260) was originally annotated as a putative phytanoyl-CoA dioxygenase (PhyH) protein due to the presence of a $\mathrm{PhyH}$ domain (Ammerman et al. 2012). PhyH or PAHX proteins in mice and humans are peroxisomal oxygenases that catalyze an essential step in the a-oxidation pathway (Wierzbicki et al. 2002). Two proteins in T. brucei contain PhyH domains, MRB7260 and a protein with TriTrypDB ID Tb927.3.3440. However, MRB7260 lacks the majority of residues that are essential for iron and 2-oxogluterate binding in mice or humans homologs (Schofield and McDonough 2007), suggesting it is catalytically inactive (Supplemental Fig. S1). Nevertheless, MRB7260 is highly conserved in trypanosomatids (Supplemental Fig. S2), consistent with its performing a conserved function. MRB7260 was absent from a high-confidence glycosome proteome of PF T. brucei (Güther et al. 2014). Rather, it reportedly associates with the T. brucei mitochondrial RNA editing machinery (Ammerman et al. 2012; Aphasizheva et al. 2014; Gazestani et al. 2016), and has been defined as a component of the mitochondrial importome by mass spectrometry analysis of mitochondria depleted for the general protein import pore TbATOM40 (Peikert et al. 2017). To confirm the mitochondrial localization of this protein, we subjected PF T. brucei cells that constitutively express MRB7260 with a myc-His-TAP-tag at its $\mathrm{C}$ terminus (MRB7260-MHT) or parental 29-13 T. brucei cells to indirect immunofluorescence. As expected, we detected a signal that colocalized with the mitochondrial marker throughout the mitochondrion for the myc-tagged MRB7260 in the MRB7260-MHT cells, but not in the 29-13 untagged control cells (Fig. 1A). Thus, MRB7260 is mitochondrially localized, as predicted for a protein associating with the mitochondrial RNA editing machinery.

The majority of proteins that associate with the mitochondrial RNA editing machinery are essential for cellular growth in PF and/or BF T. brucei (Fisk et al. 2008; Hashimi et al. 2008, 2009; Weng et al. 2008; Acestor et al. 2009; Ammerman et al. 2011, 2013; Kafková et al. 2012; Aphasizheva et al. 2014; Huang et al. 2015). To determine if MRB7260 is also important for growth, we generated $\mathrm{PF}$ and $\mathrm{BF}$ cell lines expressing tetracycline (tet) inducible RNAi against a portion of the MRB7260 open reading frame. A substantial growth defect was observed in PF RNAi-induced cells after $6 \mathrm{~d}$ with induction of tet (Fig. 1B). A modest, yet reproducible, growth defect was also seen when MRB7260 was knocked down in BF T. brucei (Fig. 1D). The reduced defect in BF may be due to the partial knockdown of the target gene in this life cycle stage. These data demonstrate that MRB7260 is essential for efficient cell growth in both PF and BF T. brucei.

To determine whether the essential phenotype of MRB7260 reflects a function of this protein in RNA editing, we examined the effect of MRB7260 repression on different classes of mitochondrial RNAs isolated from PF and BF trypanosomes. RNA levels were examined by qRT-PCR using primer sets that specifically detect either edited or pre-edited versions of each transcript (Carnes et al. 2005). Primers were also used to detect never-edited RNAs and dicistronic preprocessed (precursor) RNAs to detect effects on RNA stability or processing, respectively (Acestor et al. 2009). In PF T. brucei, edited versions of the pan-edited A6, RPS12, COIII, and ND7 RNAs were decreased to between 25\% and $36 \%$ of wild-type levels (Fig. 1C). In contrast, there was only a modest editing defect in the minimally edited RNAs (MURF2, CYb, and COII), which were decreased to $59 \%-67 \%$ of uninduced levels (Fig. 1C). The greater effect on pan-edited RNAs is similar to the trend observed when TbRGG2, a previously characterized RESC protein involved in RNA editing progression, was depleted (Fisk et al. 2008). In addition, the levels of the pre-edited versions of these RNAs were unchanged, with the exception of pre-edited COIII, which increased 2.2-fold. The lack of change in preedited RNA levels in the face of decreased edited RNAs may indicate that $3^{\prime}$ to $5^{\prime}$ progression of the editing process, rather than editing initiation, is affected. The levels of the 
A

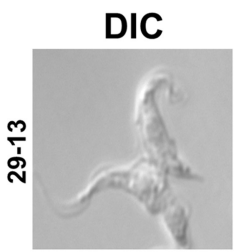

DAPI
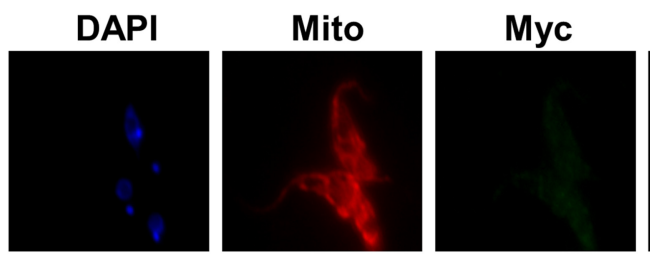

DAPI/Myc

Mito/Myc
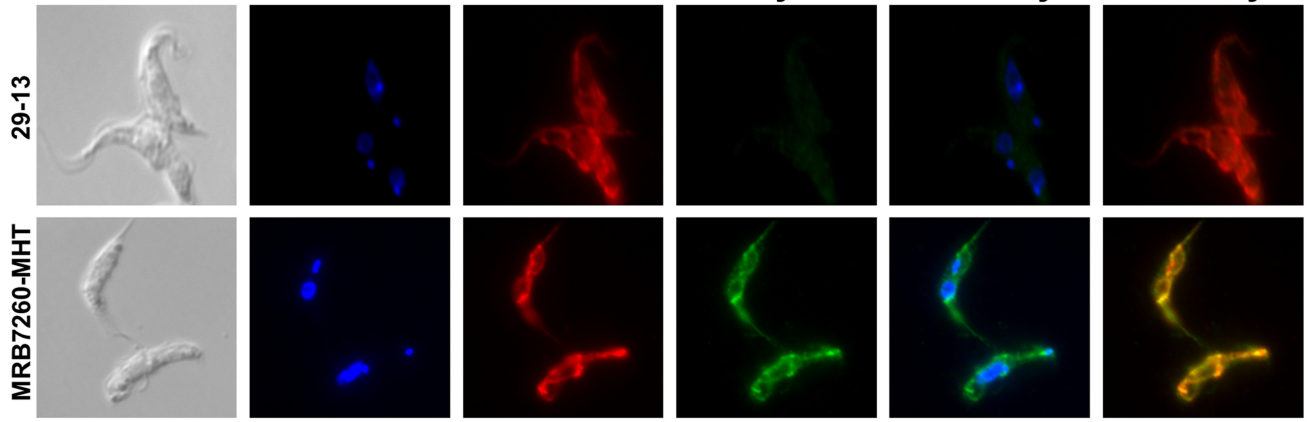

B

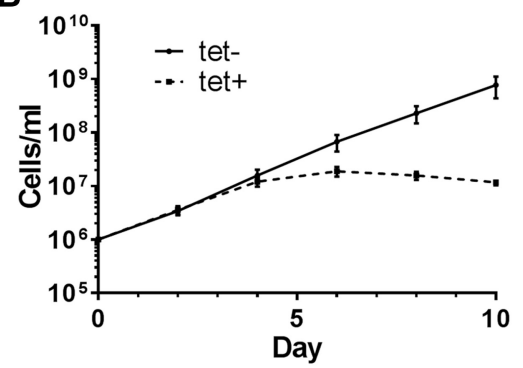

C pan-edited $\longrightarrow$ minimally-edited

D

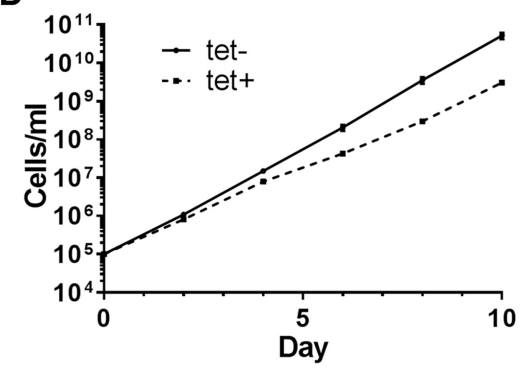

E
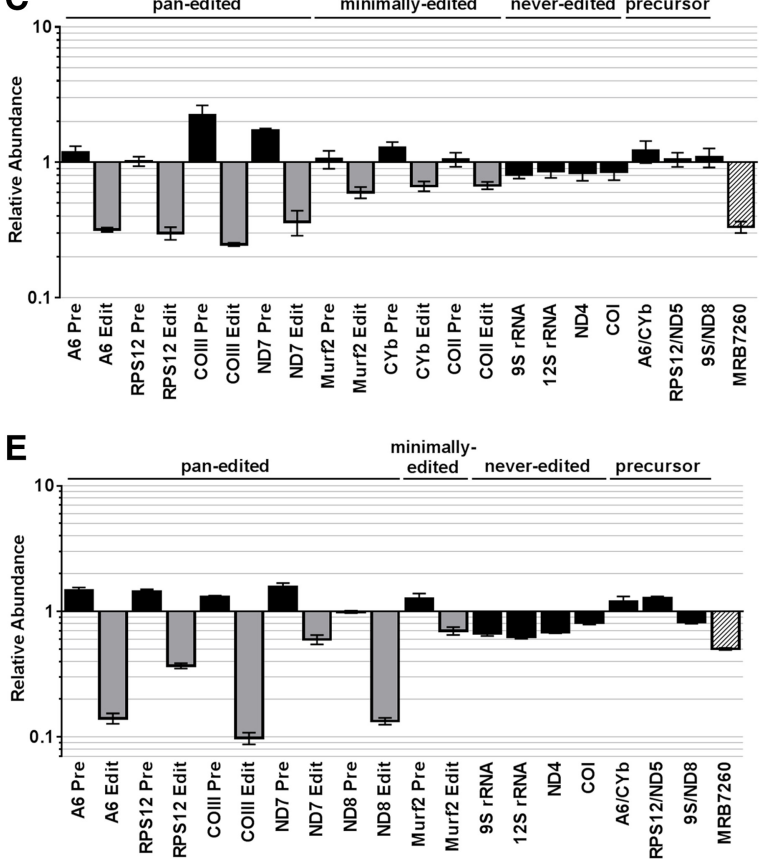

$\mathbf{F}$

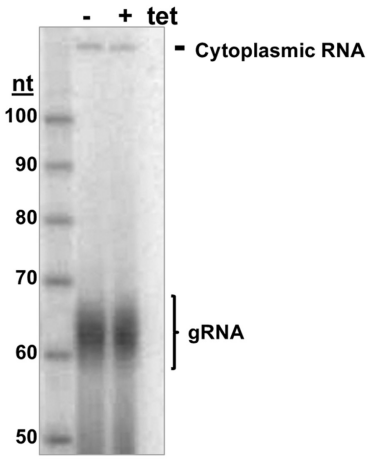

FIGURE 1. MRB7260 subcellular localization and effect of MRB7260 depletion on T. brucei growth and RNA editing. (A) PF parental 29-13 cells or MRB7260-MHT cells were used to determine the subcellular localization of MRB7260 by indirect immunofluorescence (Myc; green). Mitochondria were detected using MitoTracker Red CMXRos (Mito; red). DAPI was used to stain nuclei and kinetoplasts (blue). Signals are merged on the right (DAPI/Myc and Mito/Myc). (DIC) Differential interference contrast. (B-E) MRB7260 was repressed by tet-inducible RNAi in PF $(B, C)$ and BF $(D, E)$ T. brucei, and cell growth was measured in triplicate for uninduced (tet-) and induced cells (tet + ) for $10 \mathrm{~d}(B, D)$. RNA was isolated from procyclic $(C)$ and bloodstream $(E)$ form T. brucei on day 3 post-induction. RNA was quantified by qRT-PCR using primer sets that specifically detect never-edited, pan-edited, minimally edited, and dicistronic precursor RNAs. Relative RNA abundance represents RNA levels in tet-induced cells compared to levels in uninduced cells. RNA levels were normalized to $18 \mathrm{~S}$ rRNA levels, and numbers represent the mean and standard error of six determinations. $(F)$ RNA was isolated from PF MRB7260 RNAi cells either grown in the absence (tet-) or presence (tet + ) of tet for $3 \mathrm{~d}$ and then labeled with [ $\left.\alpha^{32} \mathrm{P}\right]-\mathrm{GTP}$ using guanylyltransferase to identify gRNAs and resolved on a denaturing gel. A cytoplasmic RNA that was used for normalization and the labeled gRNA are indicated. Biological replicate experiments were performed for $B-F$, and the level of MRB7260 knockdown was validated using qRT-PCR (PF: 26\%-40\% remaining, BF: 50\% remaining). See also Materials and Methods and Supplemental Figure S6 for an estimate of remaining protein. 
precursor transcripts spanning 9S/ND8, A6/CYb, and RPS12/ND5 and the never-edited ND4 and COI RNAs remained unaltered with repression of MRB7260, indicating the absence of any effect on RNA stability or processing (Fig. 1C). Similar trends were observed with the pan-edited, minimally edited, never-edited, and precursor RNAs when MRB7260 was repressed in BF T. brucei (Fig. 1E). Therefore, MRB7260 is required for RNA editing, with the greatest effect on pan-edited RNAs.

Next, we wanted to determine whether the inhibition of editing observed in the MRB7260 knockdown was a result of destabilization of the total gRNA population, as is in the case for GAP1/2 (Weng et al. 2008; Hashimi et al. 2009). To do so, we measured the levels of total gRNA in PF MRB7260 RNAi cells grown in the presence or absence of tet using guanylyltransferase labeling. No change was observed in the levels of gRNA between the uninduced and induced cells (Fig. 1F). It was also shown previously that knockdown of a subset of RESC proteins results in a modest accumulation of gRNA (Ammerman et al. 2013; Aphasizheva et al. 2014). Because MRB7260 depletion does not affect global gRNA levels, these data suggest a novel function for MRB720 and indicate an effect on aspects of the editing process other than gRNA stabilization.

\section{Effects of MRB7260 on RNA editing at the sequence level}

To more precisely define the step of editing that is impacted by MRB7260, we next examined the effects of MRB7260 depletion on RNA editing at the sequence population level. We used our previously described protocol that defines the regions in which the $3^{\prime}$ to $5^{\prime}$ progression of editing is paused upon depletion of essential editing factors (see Supplemental Methods; Simpson et al. 2016, 2017). Using this method, a library of pre-edited, partially edited, and fully edited sequences was obtained from two MRB7260 RNAi-induced cDNA samples and 10 uninduced control samples, two from this study and 8 from a previous study (Simpson et al. 2017). This library was aligned using TREAT (Simpson et al. 2016, 2017). TREAT defines any space between two non-T nucleotides in the cDNA as an editing site (ES), and ESs are numbered from $3^{\prime}$ to $5^{\prime}$, the direction in which overall editing progresses (Fig. 2B). An editing stop site is defined by TREAT as the $5^{\prime}$-most ES that matches the fully edited sequence, and thus any ES $3^{\prime}$ of an editing stop site matches the canonical fully edited sequence while the region $5^{\prime}$ of the editing stop site contains either pre-edited or junction sequence (Simpson et al. 2016). Junctions are variable edited sequences that match neither pre-edited nor fully edited sequence, and are present in partially edited mRNAs between the $3^{\prime}$ fully edited and $5^{\prime}$ pre-edited regions. Junctions are present in $>95 \%$ of RPS $12 \mathrm{mRNAs}$ and are hypothesized to be regions of active editing that undergo repeated remodeling (Koslowsky et al. 1991; Sturm et al. 1992; Ammerman et al.
2010; Simpson et al. 2016). TREAT designates junctions as extending from the $3^{\prime}$ most ES that does not match the fully edited sequence to the $5^{\prime}$ most editing site beyond the editing stop site that shows any editing modification. In this way, the TREAT algorithm permits analysis of junction lengths and sequence (Simpson et al. 2016, 2017).

Initially, to confirm our results from the qRT-PCR analysis in Figure 1C suggesting that MRB7260 does not affect editing initiation, we analyzed the levels of pre-edited RPS12 mRNAs in our high-throughput libraries. The number of normalized pre-edited transcripts for the uninduced and MRB7260 RNAi-induced samples were averaged and plotted in Figure 2A. Similar to the results of qRT-PCR (Fig. 1C), we observed no effect on the level of RPS12 pre-edited transcripts when MRB7260 was depleted (Fig. 2A), indicating that MRB7260 is not required for editing initiation. Because MRB7260 depletion leads to a decrease in fully edited mRNA in the absence of corresponding pre-mRNA accumulation, the protein is likely required for the $3^{\prime}$ to $5^{\prime}$ progression of editing. Thus, we next asked whether MRB7260 depletion has a greater effect on gRNA exchange or on the progression of editing through gRNA-defined regions. The gRNA-directed regions are based on a recent gRNA transcriptome study (Koslowsky et al. 2014), and we recently provided evidence that the positions of these gRNA families are conserved between strains (Simpson et al. 2017). Using TREAT analysis, we determined the ESs at which editing stalled upon depletion of MRB7260. Editing stop sites that significantly increased $(\mathrm{p}<0.05, \mathrm{q}<0.05)$ upon the depletion of the protein were termed exacerbated pause sites (EPSs) (Supplemental Table S1; Simpson et al. 2017). EPSs are comprised of sequences that share the same $5^{\prime}$-most site of correct editing, yet may vary in junction sequence and length. Figure 2B shows RPS12 mRNA aligned with its cognate gRNAs (Koslowsky et al. 2014), which are numbered sequentially from $3^{\prime}$ to $5^{\prime}$. As indicated by black diamonds in Figure 2B, we observed numerous EPSs throughout the lengths of gRNAs when MRB7260 is depleted. The average number of sequences for uninduced and induced samples at each MRB7260 EPS is shown in Figure 2C. Within RPS12 mRNA, EPSs arising from MRB7260 depletion occur in clusters within each gRNA block from gRNA 2-7, indicating the requirement of the protein for the progression of editing through gRNA-defined regions. The absence of EPSs in the first gRNA-directed block is also consistent with MRB7260 playing no role in initiation of the editing process, but rather being needed for later editing stages.

Several REMC proteins (TbRGG2, MRB8180, and MRB8170/4160) were recently examined for their roles in the progression of editing (Simpson et al. 2017), and though MRB7260 plays a role in editing progression, several pieces of evidence suggest that its role is distinct from these previously examined REMC factors. Strikingly, the lack of EPSs within the first gRNA-directed block distinguishes MRB7260 from the REMC proteins. Previously determined EPSs resulting 


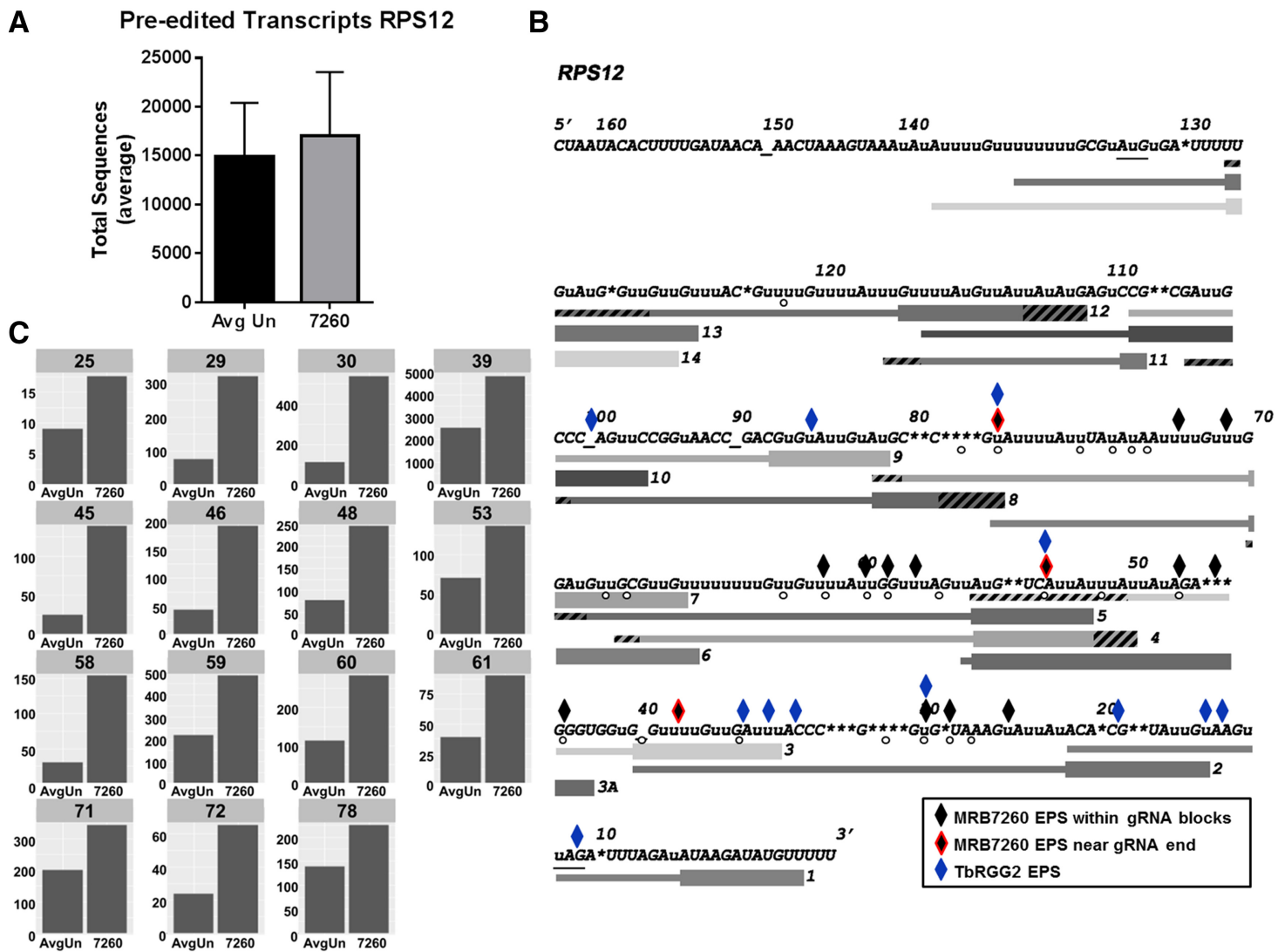

FIGURE 2. Analysis of RPS12 transcripts from MRB7260 replete and depleted cells at the sequence level using TREAT. (A) The average number of normalized pre-edited RPS12 transcripts for the 10 uninduced samples (Avg Un), and two MRB7260 RNAi-induced samples (7260) in PF T. brucei. (B) RPS12 edited mRNA sequence with the exacerbated pause sites (EPSs) that arise upon depletion of MRB7260 for both biological replicates indicated with black diamonds. Solid black diamonds represent MRB7260 EPSs within the gRNA-directed region, whereas black diamonds with red outlines indicate MRB7620 EPSs near gRNA ends. Blue diamonds show EPS from a previous study (Simpson et al. 2017) for the knockdown of TbRGG2. Gray bars below the sequences display regions guided by specific gRNAs as reported previously (Koslowsky et al. 2014) and are numbered from the $3^{\prime}$ to $5^{\prime}$ direction along the mRNA. gRNA anchor regions at the $5^{\prime}$ of the gRNA are depicted with bold lines, whereas the hatched regions at the $5^{\prime}$ and $3^{\prime}$ ends represent variation in gRNA lengths within that class of gRNA. The start and stop codons of the coding sequence are underlined. Open circles indicate editing stop sites in which junction length zero is significantly increased when MRB7260 is depleted in both replicates compared to the average of the uninduced samples. Underscores are shown for clarity in stretches of unedited sequence so that numbers above align with the correct ES. (C) Average number of RPS12 transcripts for the 10 uninduced (Avg Un) and the two MRB7260 RNAi-induced (7260) samples at each EPS for the MRB7260 knockdown.

from TbRGG2 depletion are shown by blue colored diamonds for comparison in Figure 2B (Simpson et al. 2017). Additionally, the positioning of EPSs in regions defined by gRNA 2-7 also differs between MRB7260-depleted cells and those that are depleted of TbRGG2. While the latter were concentrated in regions directed by the $3^{\prime}$ ends of gRNAs, EPSs in MRB7260-depleted cells are observed primarily in clusters located in regions corresponding to more $5^{\prime}$ regions of gRNAs (e.g., ES 25, 29, and 30; ES 45, 46, and 48; ES 58-61; ES 71 and 72), suggesting an involvement of MRB7260 in early stages of gRNA-mRNA positioning. In addition to EPSs that occur within gRNA-directed blocks, we also observed three MRB7260 EPSs arising near the end of gRNAs (E3S9, ES53, and ES78; black diamonds with red outlines; Fig. 2B). The positions of the EPSs caused by depletion of MRB7260 suggest this protein is functionally distinct from REMC factors and support a role for MRB7260 in
gRNA utilization and gRNA exchange, after the initial gRNA-directed block.

As the above data suggest that MRB7260 plays a role in gRNA utilization and gRNA exchange, we next examined the sequences of junctions arising $5^{\prime}$ of EPSs in MRB7260-depleted cells to gain insights into the specific functions of MRB7260. We undertook a detailed examination of the most abundant editing intermediates in MRB7260-depleted cells, focusing on the second gRNA-directed editing block of RPS12 mRNA, which spans ESs 22-40 (Fig. 3). Shown are RPS12 junction sequences for which there were at least 100 average normalized counts in the MRB7260 RNAi samples. The sequences were divided between the two apparent functions of MRB7260: progression through the gRNA (EPSs throughout the gRNA-2 defined region, Fig. 3A) and gRNA exchange (EPSs at the $3^{\prime}$ end of gRNA-2, Fig. 3B). For each sequence, the average normalized count in the 


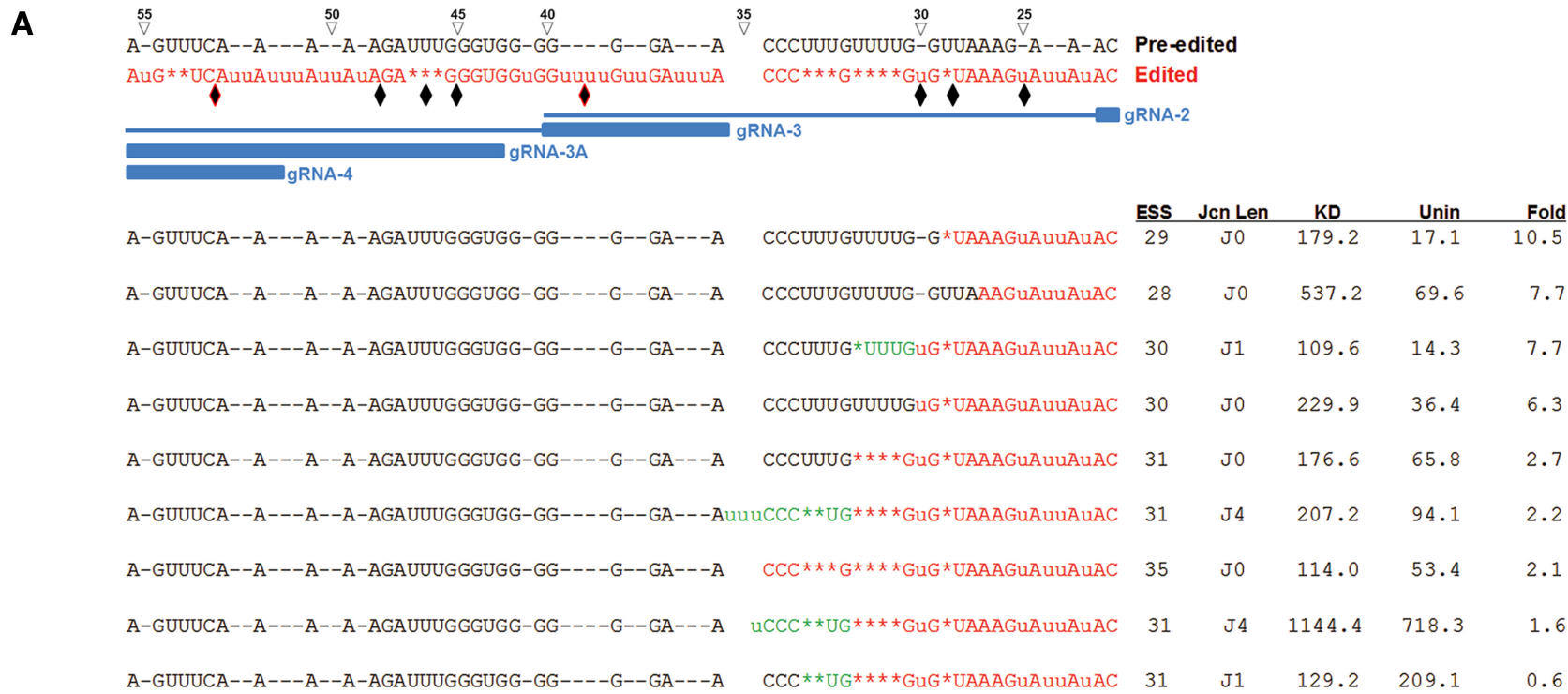

\section{B}

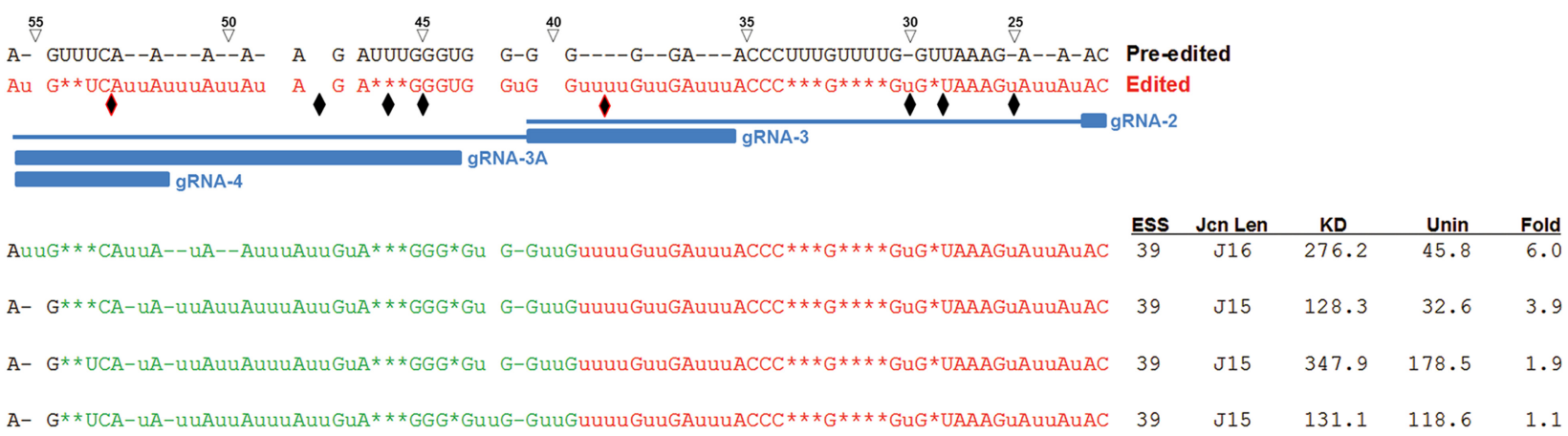

FIGURE 3. Sequence analysis of the most abundant junctions that occur within the second gRNA block MRB7260 replete and depleted cells. Average number (normalized counts) of junction sequences in the uninduced samples (Unin) and the MRB7260 knockdown (KD) from editing stop sites within the second gRNA guided block (editing stop sites 22-40). ESS refers to the editing stop site for that sequence, whereas Jcn Len indicates the number of editing sites that the junction sequence spans. Fold indicates the fold change in the number of sequences for each specific junction in the MRB7260 RNAi samples compared to the uninduced samples. Pre-edited sequences are displayed in black, edited RNA is indicated in red, while junctions are shown in green. Blue lines below the pre-edited and edited transcripts show the gRNA-directed blocks. The positions of the ES are indicated with triangles and are numbered from $3^{\prime}$ to $5^{\prime}$. EPSs that arise following MRB7260 depletion are shown with black diamonds, with sites within the gRNA-directed blocks (all black) or near gRNA ends (black with red outline) as in Figure 2. The most abundant junction sequences within the gRNA-2 directed block are listed and grouped according to those that indicate a defect in editing progression $(A)$ or correct gRNA exchange $(B)$ when MRB7260 is depleted.

MRB7260 RNAi samples was compared to that of the average normalized count in the uninduced samples to calculate the fold change in sequence abundance when MRB7260 is depleted, and sequences are presented in order of decreasing fold change.

Within the region guided by gRNA-2, we observed a twoto 10 -fold increase in sequences lacking a junction (denoted junction length zero or J0) at editing stop sites 28-31 and 35 (Fig. 3A). These sites represent sequential, correct editing modifications moving $3^{\prime}$ to $5^{\prime}$ along the gRNA-2 guided region. In addition, there was an increase in sequences with short junctions at editing stop sites 30 and 31 ranging from 1.6-7.7-fold when MRB7260 was depleted, with the greatest increases in the shortest junctions (length 1) (Fig. 3A). A pre- vious study examining RNA editing progression within the gRNA-1 directed block of RPS12 showed that an increase in sequential junction zero sequences represented a shift from nonlinear editing progression to a linear $3^{\prime}$ to $5^{\prime}$ site by site modification (Simpson et al. 2017). In this previous study, editing within the gRNA-1 directed block of RPS12 progressed approximately in a 50\% nonlinear and 50\% linear fashion in the uninduced samples (Simpson et al. 2017). In contrast, early editing within the gRNA-2 directed block of RPS12 progresses almost completely in a linear manner even in RNA from the uninduced cells (data not shown). Therefore, we conclude that the increase in junction zero sequences upon MRB7260 depletion represents a buildup of the normal editing intermediates early during gRNA-2 
directed editing. Looking at changes in zero junction length sequences, we observed that while sequences with no junctions were not significantly increased across the entire transcript (Supplemental Fig. S3A), sequences lacking junctions were significantly increased $(\mathrm{p}<0.05, \mathrm{q}<0.05)$ at specific editing stop sites that commonly had short or no junction sequences in the uninduced samples (Fig. 2B, open circles; Supplemental Fig. S3B,C). Many of these sites were early in a gRNA-directed region, consistent with the observations in gRNA-2. Additionally, of the 15 EPSs in MRB7260 RNAi cells, eight, including the sites early in gRNA-2, showed a significant increase in junction length zero (Fig. 2B, all black diamonds with open circles). Thus, the dramatic buildup of junction zero sequences in gRNA-directed regions just following the anchor sequence supports a role for MRB7260 in gRNA positioning early in the utilization of a given gRNA. These data further support the conclusion that MRB7260 plays a distinct role compared with previously studied REMC factors.

When examining junction sequences at EPSs in the second gRNA-directed editing block of RPS12 mRNA in MRB7260 RNAi cells, we also observed four abundant junction sequences that did not occur in the middle of the gRNA, but rather arose at ES39 (an EPS in the MRB7260-depleted sample), which abuts a gRNA end (Fig. 3B). Three of these four sequences exhibit an increase, between two- and sixfold, upon MRB7260 depletion. Interestingly, the junction sequences arising at this apparent gRNA end are substantially longer than those arising within the gRNA-directed block, spanning between 15 and 16 ESs (Fig. 3B), compared to zero to 4 ESs within the gRNA-directed block (Fig. 3A). Moreover, the junctions in those sequences that increase in MRB7260 RNAi cells are identical at their first 10 ES. The positioning and sequences of these junctions suggests that they may have arisen due to utilization of an alternative gRNA. This finding is in contrast to what we previously observed upon depletion of GAP1, where we did not see a dramatic increase in these sequences, but rather observed sequences with no junction one site further $5^{\prime}$ (ES40) at the proposed end of gRNA-2 (Simpson et al. 2017). Because this specific sequence family increases in MRB7260-depleted cells (Fig. 3B), these data implicate MRB7260 in proofreading correct gRNA-mRNA contacts during gRNA exchange. Thus, in both the progression of editing through a gRNA and in gRNA exchange, MRB7260 plays a role that differs from both previously studied REMC factors and GAP1.

\section{MRB7260 interacts with multiple components of the RESC complex}

MRB7260 has been reported in pulldowns of the RESC complex (Ammerman et al. 2012; Aphasizheva et al. 2014). Thus, we next wanted to better define its interactions with the RNA editing machinery to further illuminate its role it the editing process and determine the biochemical basis of the sequence level effects described above. To begin, we used the MRB7260 RNAi cell line to ask whether the presence of MRB7260 affects the steady-state levels of proteins involved in RNA editing. Cell lysates were probed with antibodies against multiple RESC components that partition with either the GRBC or REMC subcomplexes, as well as a component of the RECC complex, KREPA6. The steady-state levels of the RESC or RECC proteins examined are unaltered (Fig. 4A). Thus, MRB7260 depletion does not impact RESC or RECC protein abundance.

To determine the direct protein-protein interactions of MRB7260, we utilized a yeast two-hybrid assay. Previously, a comprehensive yeast two-hybrid screen was performed to determine direct protein-protein interactions between components of the RESC complex (Ammerman et al. 2012). However, MRB7260 was not included in this screen. A summary of the results from the previous yeast two-hybrid study (Ammerman et al. 2012) and this study is shown in Figure $4 \mathrm{~B}$. For the yeast two-hybrid analysis, interactions were categorized as strong (MRB3010-AD and MRB8620-BD, shown as $[+++++]$ ) or weak (MRB11870-AD and GAP1$\mathrm{BD}$, shown as $[+++])$, using interactions from the previous screen for comparison, as seen in the representative plates in Figure 4C. Using this scoring system, we tested the interaction of MRB7260 with components of GRBC (MRB3010, MRB11870, GAP1, GAP2, MRB5390, MRB8620, MRB0880) or REMC (TbRGG2, MRB8170, MRB4160, MRB8180, MRB800, MRB1860) and MRB10130. Empty vectors were also used as a negative control. Of all the interactions tested, MRB7260-AD displayed only weak, but reproducible, interactions with GAP1-BD, MRB8620-BD, and MRB8170-BD (Fig. 4B,C). There were no interactions observed when MRB7260 was fused to the BD. An absence of an interaction in the yeast two-hybrid screen may represent the inability of these proteins to interact in vivo. However, false negatives may also arise due to an RNA-dependence of the protein interaction between MRB7260 and the RESC components, poor protein expression, or the presence of the $\mathrm{AD}$ or $\mathrm{BD}$ tag interfering with interactions in this system. From these results, we conclude that MRB7260 has weak protein interactions with two GRBC components, GAP1 and MRB8620, and REMC component MRB8170.

To confirm the protein-protein interactions observed by the yeast two-hybrid screen and possibly identify additional interactions, we used the PF T. brucei cell line containing the tagged MRB7260-MHT. In vivo IPs were performed in the presence of RNA (lysate treated with RNase inhibitor) or absence of RNA (lysate treated with an RNase cocktail) to distinguish RNA-inhibited and RNA-enhanced interactions. We used available antibodies to detect proteins that copurified with MRB7260 using western blot. Two proteins, GAP1 and MRB8170, displayed an RNA-enhanced interaction with MRB7260 as shown in Figure 4D, where down arrows indicate a decrease in association with MRB7260 when RNA is removed. We note that these are two proteins 
A

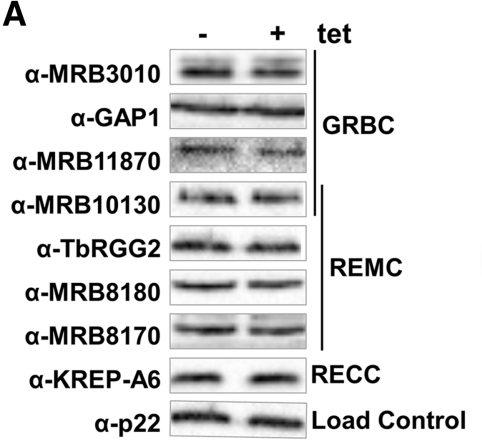

B RNA Editing Substrate Binding Complex (RESC)

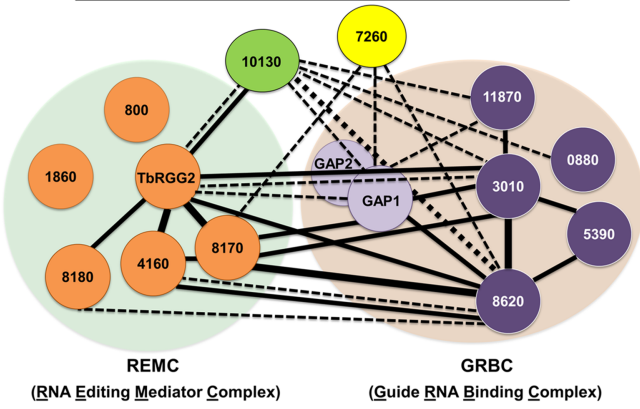

D

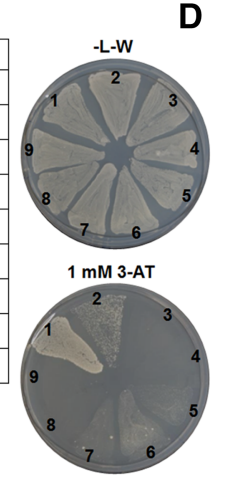

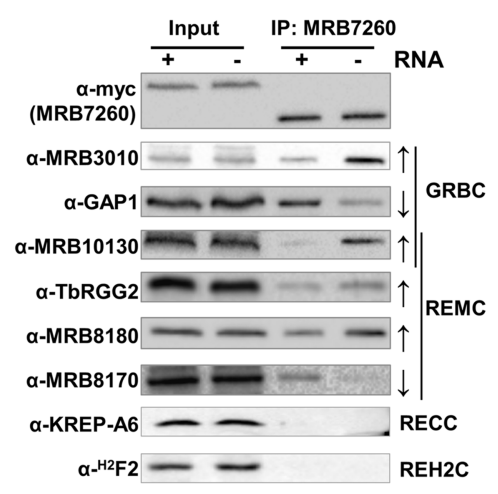

FIGURE 4. MRB7260 protein-protein interactions. (A) PF T. brucei cells harboring the MRB7260 RNAi construct were grown for $3 \mathrm{~d}$ in the presence or absence of tet. Cell lysates were then analyzed by western blot using antibodies for components of the GRBC subcomplex, the REMC subcomplex, and RECC. Western blot using an antibody against p22 served as a load control. MRB7260 knockdown was detected by qRT-PCR ( 20\% of wild type levels). Figure shown is representative of two technical replicates. (B) Schematic representation of the RESC complex divided into the GRBC and REMC subcomplexes. Solid lines (strong) or dotted lines (weak) indicate the strength of interaction observed in a published yeast two-hybrid screen (Ammerman et al. 2012) and with MRB7260 performed in this study. Interactions observed in both directions are shown using thick lines, whereas thin lines represent interactions that occurred in one direction of the screen. These direct protein-protein interactions were verified using immunoprecipitation (IP) to place each protein into either the REMC or GRBC subcomplexes (Ammerman et al. 2012, 2013). (C) A subset of the direct protein interactions tested using a yeast two-hybrid screen. pGAD-T7 is a vector used to express prey activation domain fusion proteins, while pGBK-T7 expressed the bait binding domain fusion proteins. Yeast cells were grown on media lacking leu and $\operatorname{trp}(-\mathrm{L}-\mathrm{W})$ to select for cotransformants and then subsequently grown on plates lacking leu, trp, and his but supplemented with $1 \mathrm{mM} 3$-AT to select for bait-prey interactions. Interactions were then scored according to previously described strong (MRB3010-MRB8620, +++++ ) or weak (MRB11870-GAP1, +++) interactions (Ammerman et al. 2012). Results are representative of at least two biological replicates for each interaction. (D) MRB7260-MHT (Myc-His-TAP) and associated proteins were affinity purified from PF T. brucei cell extracts that were either RNase inhibited (RNA+) or RNase treated (RNA-). Proteins were eluted from IgG beads using TEV protease cleavage and electrophoresed on $10 \%$ (w/v) SDS-PAGE gels, followed by western blotting using antibodies against myc (to detect MRB7260) and various mitochondrial proteins. For visualization, $0.025 \%$ of input and $1 \%$ of TEV elutions were loaded. Arrows indicate the change in protein association with MRB7260 when RNA is removed. Representative of two biological replicate experiments.

that weakly interacted with MRB7260 by yeast two-hybrid assay (Fig. 4C). In contrast, multiple proteins interacted with MRB7260 in a manner that was inhibited by RNA (Fig. 4D, up arrows indicate increased interaction with RNA is removed). MRB3010 and MRB10130 show a dramatic increase in association when RNA is removed using RNase treatment, while TbRGG2 and MRB8180 display a modest increase (Fig. $4 \mathrm{D}$, up arrows). Interestingly, proteins previously proposed to be components of a single subcomplex, either REMC or GRBC, display differential association with MRB7260. For example, both MRB8180 and MRB8170 are depicted as REMC components; however, MRB8180 exhibits an RNA-inhibited interaction with MRB7260 while an RNA-enhanced interaction is observed for MRB8170 (Fig. 4D). Likewise, the interactions of GAP1 and MRB3010, typically depicted as GRBC components, with MRB7260 have opposite RNA sensitivities (Fig. 4D). This leads us to conclude that MRB7260 does not associate with proteins within one subcomplex in the same manner. In addition to RESC components, we also tested interaction of MRB7260 with KREPA6, which is a RECC complex protein, but no interaction was detected (Fig. 4C). ${ }^{\mathrm{H} 2} \mathrm{~F} 2$, a component of the $\mathrm{REH} 2 \mathrm{C}$ complex that promotes RNA association with RESC (Madina et al. 2014; Kumar et al. 2016), also did not interact with MRB7260 (Fig. 4D). Therefore, MRB7260 associates with components of both the REMC and GRBC subcomplexes in a manner affected by RNA, but does not stably interact with RECC or the REH2C complex.

Because interactions between MRB7260 and the editing machinery are impacted by RNA, we asked whether MRB7260 itself exhibits RNA binding activity. To this end, we performed in vitro UV crosslinking assays with body-labeled gA6[14] gRNA (Read et al. 1994) or a 79-nt A6U5 pre-mRNA (Fisk et al. 2008) and recombinant MRB7260 (Supplemental Fig. S4A). We did not detect RNA binding activity using this assay, although CD spectra of recombinant MRB7260 indicated that the protein was folded (Supplemental Fig. S5). We next tested the RNA binding activity of MRB7260 by UV crosslinking in vivo followed by limited RNase digestion and RNA endlabeling, but this assay also failed to reveal any RNA binding activity of MRB7260 (Supplemental Fig. S4B). These data suggest that MRB7260 does not directly bind RNA. However, we cannot rule out the ability of this protein to bind RNAs other than those tested or a failure to undergo UV crosslinking.

The differential association of MRB7260 with RESC proteins from the same subcomplex (Fig. 4D) led us to hypothesize that MRB7260 is interacting with these proteins 
A
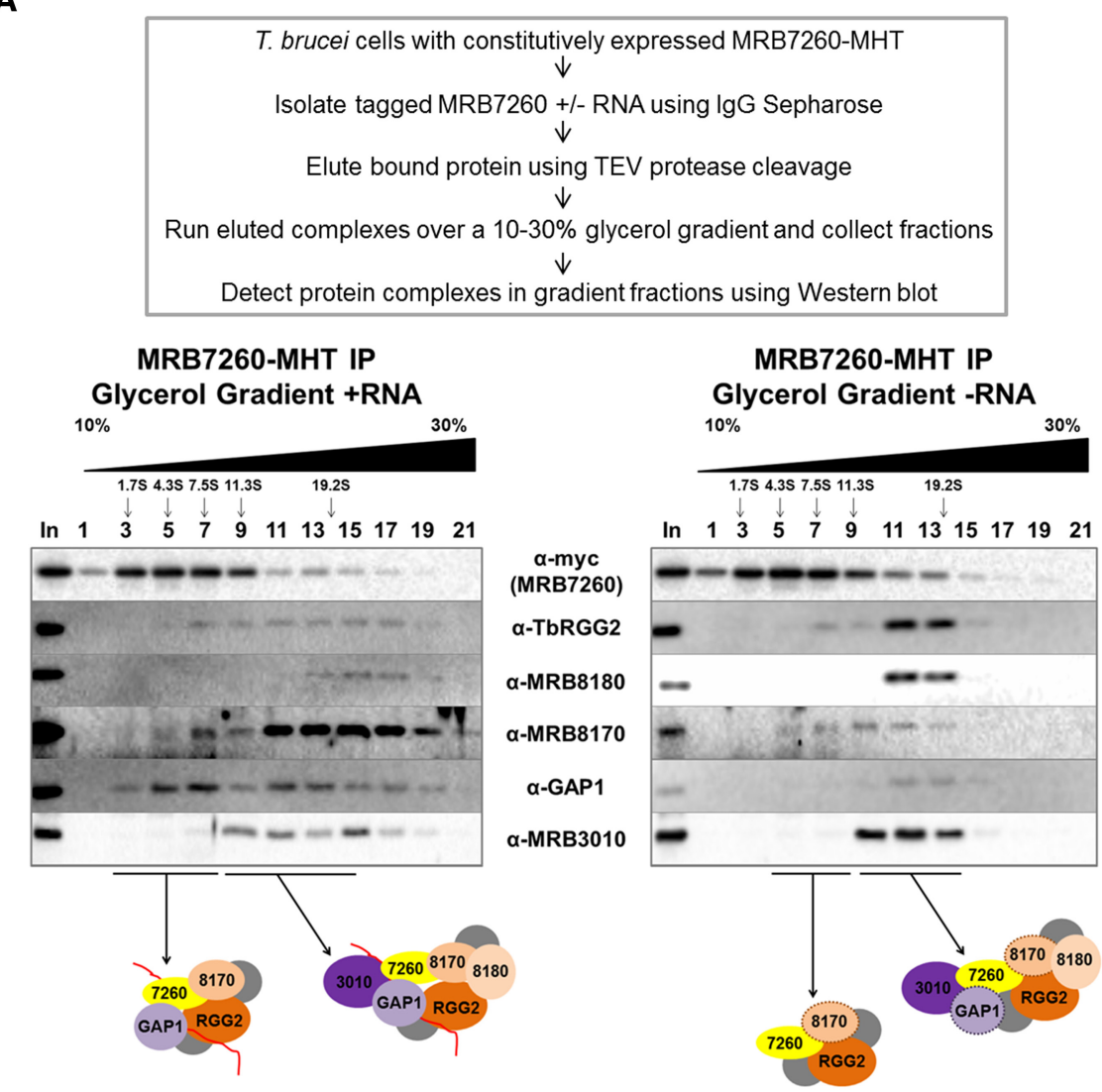

B

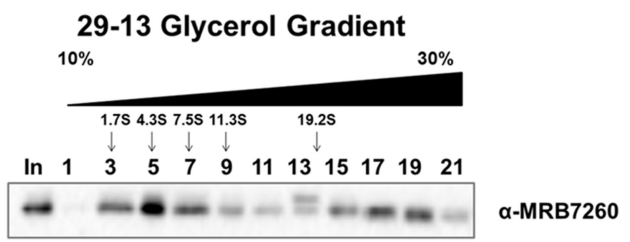

FIGURE 5. Visualization of MR7260 complexes using gradient sedimentation. (A, top) Stepwise description of the method used to visualize the MRB7260-containing complexes. (Bottom) MRB7260-MHT was affinity purified from PF T. brucei in the presence or absence of RNA and then subjected to $10 \%-30 \%(\mathrm{v} / \mathrm{v})$ glycerol-gradient sedimentation. Fractions collected were analyzed by western blot with antibodies against RESC proteins. Size markers obtained from a parallel gradient are displayed above the fraction numbers. Representative western blots of two biological replicate experiments. Schematic representation of the proteins found in the fractions is displayed below the blots. Dotted borders around the proteins indicate a decreased presence of the protein in a given fraction when RNA is removed. Gray circles indicate unknown proteins that may also be present within these complexes. Pictures show the presence or absence of proteins and are not meant to indicate stoichiometry. (B) Total cell lysate (not treated with RNase) from the parental PF T. brucei strain 29-13 cell line was subject to $10 \%-30 \%$ (v/v) glycerol gradient sedimentation. Fractions were collected and analyzed by western blot with antibodies against MRB7260. Shown is a representative of two biological replicate experiments.

in separate MRB7260-containing complexes. To visualize different MRB7260-RESC protein complexes that occur in the presence or absence of RNA, TEV elutions of MRB7260 pulldowns (Fig. 4D) were subjected to centrifugation over $10 \%-30 \%$ glycerol gradients, fractions were collected, and the proteins present were detected by western blot with available antibodies (Fig. 5A). In samples in which RNA was preserved, we detected multiple MRB7260-containing complexes (Fig. 5A, bottom left). The majority of MRB7260 peaks in fractions $3-7$, and these fractions clearly co-peak with GAP1, indicating the existence of a particle containing these two proteins. A peak of MRB8170 is present in fraction 7 , and TbRGG2 is also present, suggesting that MRB7260/GAP1 complexes can contain one or both of these proteins. We also observe a larger complex(es) that contains MRB3010 and MRB8180, perhaps representing fully assembled RESC (Fig. 5A, bottom left, fractions 9-19). When RNA is removed from the lysate prior to IP, we observe a loss of the MRB7260-associated GAP1 and MRB8170 (Fig. 5A, bottom right), in keeping with the RNA-enhanced interactions shown in Figure 4D. The larger MRB7260-containing complexes are shifted to fractions 9-13, presumably due to loss of both RNA and protein. To determine if the small MRB7260-containing complexes that peak in fractions 3-7 represent native complexes, we generated antibodies against recombinant MRB7260 to probe glycerol gradient fractions generated from the parental T. brucei cell line, strain 29-13. Similar to the tagged protein (Fig. 5A, bottom left), native MRB7260 displayed a peak in fractions 3-7, along with a second peak in fractions 17-19 (Fig. 5B). From these data, we conclude that MRB7260 not only interacts with the assembled RESC complex, but that abundant smaller complexes containing at least MRB7260 and GAP1 are present.

\section{MRB7260 affects the protein associations between the GRBC and REMC subcomplexes}

Having shown that MRB7260 interacts with RESC proteins that are components of both the GRBC and REMC subcomplexes, we next wanted to determine if depletion of MRB7260 affects subcomplex interactions. To do so, we utilized a PF $T$. brucei cell line containing both the MRB7260 RNAi construct and the constitutively tagged TbRGG2-HTM (His-TEV-myc) to perform IPs of the REMC subcomplex in the presence or absence of MRB7260. When MRB7260 was depleted, the association of TbRGG2 with other REMC components, MRB8180 or MRB8170, was unchanged (Fig. 6A,B). However, association 
A
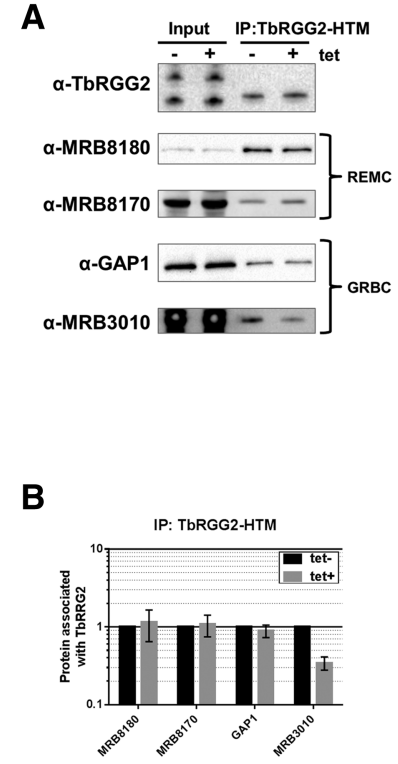

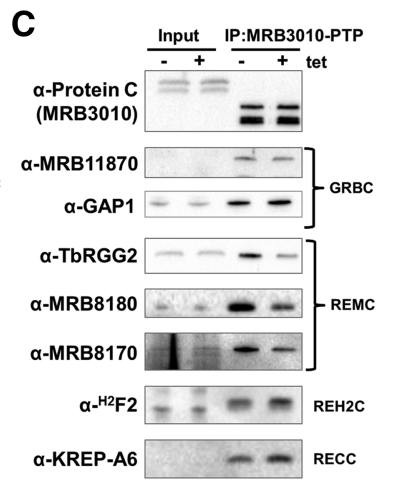

D

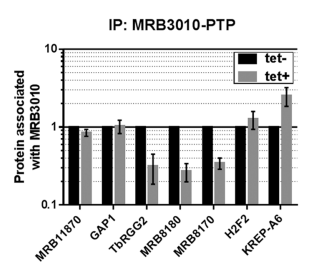

FIGURE 6. Effect of MRB7260 depletion on RESC protein-protein interactions. (A) PF T. brucei cells harboring both the MRB7260 RNAi construct and the TbRGG2-HTM construct were used to assess the proteins associated with the REMC subcomplex in the absence of MRB7260. Cells were grown for $3 \mathrm{~d}$ in the absence or presence of tet, followed by IP of TbRGG2 using the myc tag. Bound protein was eluted from myc beads using TEV protease cleavage. Elutions were then analyzed using western blot with a subset of REMC and GRBC protein antibodies. Western blots display $0.025 \%$ of input and $6 \%$ of TEV elution. Biological replicate experiments were performed and qRT-PCR was used to validate the level of MRB7260 knockdown (29\%-44\% remaining). (B) Quantification of western blots in $A$ using BioRad Image Lab software. Protein levels were normalized to amount of TbRGG2 for that immunoprecipitation (IP). The protein associated with TbRGG2 was then calculated by comparing the normalized protein levels from the tet+ IP to that of the tet- IP (which was set to 100\%). Bar graphs represent the average and standard deviation of two biological replicates with a total of $n=4$ for each antibody. (C) PF T. brucei cells containing both the MRB7260 RNAi construct and the MRB3010-PTP construct were used to identify proteins associated with GRBC when the levels of MRB7260 are altered. Cells were either induced with tet or left uninduced for $3 \mathrm{~d}$ followed by IP of MRB3010 using IgG. Protein elutions that resulted from TEV protease cleavage were then analyzed using western blot, with $0.015 \%$ of input and $1 \%$ of TEV elution loaded. Biological replicate experiments were performed and qRT-PCR was used to validate the level of MRB7260 knockdown (50\%). (D) Quantification of western blots in $C$ using BioRad Image Lab software. Protein levels were normalized to the amount of MRB3010 for that IP. The protein associated with MRB3010 was then calculated by comparing the normalized protein levels from the tet+ IP to that of the tet- IP (which was set to $100 \%)$. Bar graphs represent the average and standard deviation of two biological replicates with a total $n=4$ for each antibody.

with MRB3010, which is a core component of the GRBC subcomplex, was decreased to $\sim 35 \%$ of the level in the uninduced IP. Therefore, without MRB7260 there is a decreased association between the REMC and GRBC subcomplexes. Interestingly, the association of GAP1 with TbRGG2 was unaltered with the depletion of MRB7260 (Fig. 6A,B), again indicating that MRB7260 interacts differentially with distinct components of GRBC and that multiple GAP1 pools may be

present. To further show that MRB7260 affects REMCGRBC interactions, we generated a PF T. brucei cell line that contains the MRB7260 RNAi construct in addition to a constitutively expressed PTP-tagged MRB3010 (Ammerman et al. 2011) to IP the GRBC subcomplex in the presence or absence of MRB7260. As with the REMC complex described above, depletion of MRB7260 did not affect the GRBC intracomplex protein interactions (MRB11870 and GAP1). However, the GRBC-REMC interactions (TbRGG2, MRB8180, and MRB8170) were decreased in MRB7260-depleted cells (Fig. 6C,D). This decreased REMC-GRBC interaction was specific, because the interactions between GRBC and either the REH2C complex or RECC were unaffected or slightly increased, respectively (Fig. 6C,D). These data demonstrate that MRB7260 stabilizes the interaction between REMC and GRBC.

\section{RNA association with RESC is altered with depletion of MRB7260}

Because depletion of MRB7260 alters the protein-protein association between the subcomplexes of RESC (Fig. 6), we next asked whether MRB7260 impacts RNA associations with these subcomplexes. We again utilized the PF T. brucei cell lines containing both the MRB7260 RNAi construct and either the MRB3010-PTP or TbRGG2-HTM construct to perform RNA immunoprecipitations (RIPs). RNA was measured using $\mathrm{qRT}$-PCR with primers that detected a subset of pan-edited mRNAs and gRNAs. In order to detect the largest pool of mRNA for a specific transcript, we designed primers that hybridized to the $5^{\prime}$ never-edited region and a portion of the $5^{\prime}$ pre-edited sequence of several mRNAs. These primers will detect the entire pool of pre-edited and partially edited versions of a given transcript, with the exception of those that are edited in the extreme $5^{\prime}$-most region (Fig. 7A). Because only a small fraction of total mRNA is edited this far $5^{\prime}$ (Simpson et al. 2016, 2017), depletion of MRB7260 does not affect the amount of mRNA detected by these primer sets (Fig. 7B). Therefore, RIP data are not confounded by large changes in the input levels of a given mRNA (Madina et al. 2014; Huang et al. 2015; Kumar et al. 2016). A series of primers that detect specific gRNAs were also designed. To begin, we tested whether the target RNAs are enriched in TbRGG2-HTM pulldowns compared to a mock IP, and showed that a subset of mRNAs and gRNAs are two- to 30-fold enriched (Fig. 7C). We then asked whether MRB7260 affects the levels of RNAs associated with the REMC subcomplex by comparing RNAs in TbRGG2-HTM pulldowns either replete for or depleted of MRB7260. We observed a five- to 10 -fold increase in mRNA associated with REMC when MRB7260 was depleted compared to the uninduced RIP (Fig. 7D). In addition, there was also a two- to fivefold increase in four of the five gRNAs detected with the complex when MRB7260 was depleted (Fig. 7D). Therefore, removal of MRB7260 results in an increased 
A

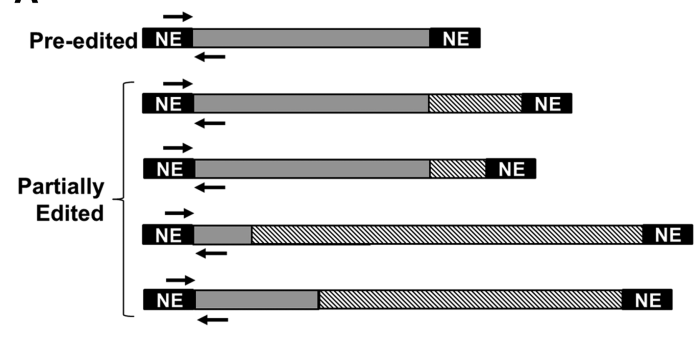

Fully Edited NE

C

E
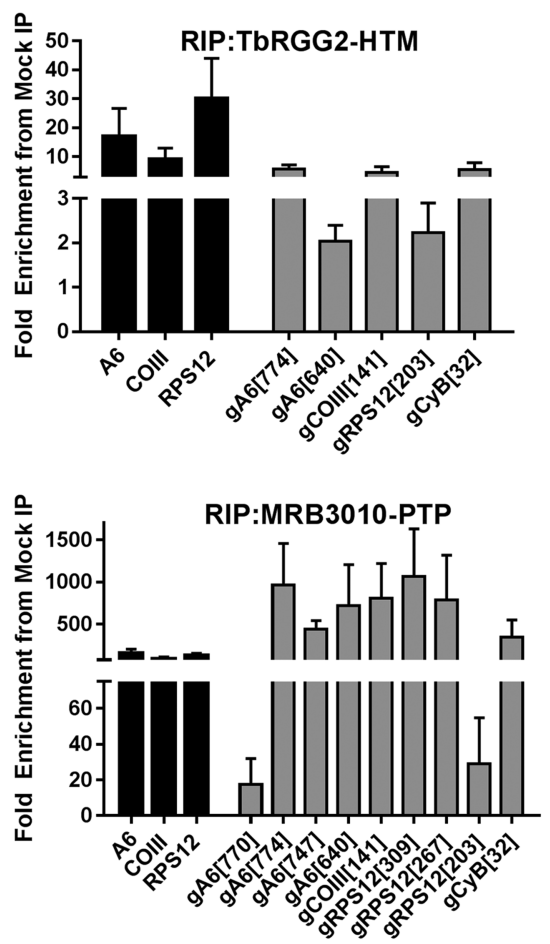

B

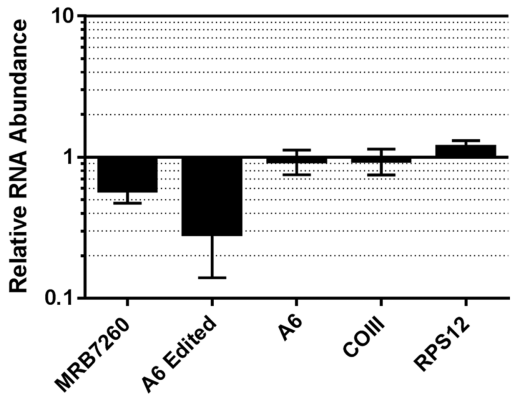

D
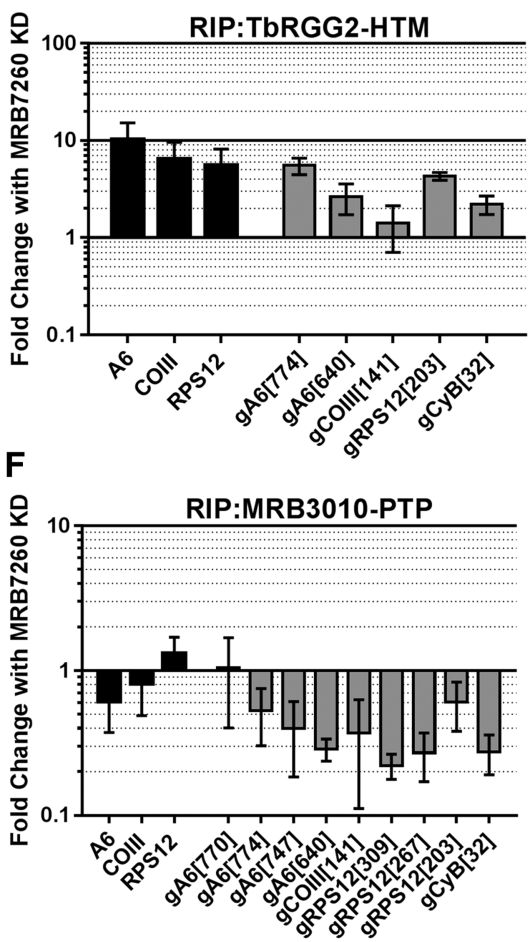

FIGURE 7. Effect of MRB7260 depletion on RESC protein-RNA interactions. (A) Schematic representation of the qRT-PCR primers used to detect the largest pool of mRNAs for a given transcript in RIPs. Pre-edited regions are indicated in gray and edited RNA is displayed using hashed lines. RIP mRNA primers hybridize to the $5^{\prime}$ never-edited (indicated in black) and $5^{\prime}$-most pre-edited region of the transcripts. RIP primers detect pre-edited and all partially edited RNAs, excluding RNAs that are fully edited. (B) Total cellular RNA from PF cells containing the MRB7260 RNAi construct grown with or without tet for $3 \mathrm{~d}$ were analyzed using the RIP qRT-PCR primers. Relative RNA abundance represents RNA levels in tet-induced cells compared to uninduced. RNA levels were normalized to $18 \mathrm{~S}$ rRNA levels. For comparison, results with primers that detect only fully edited A6 mRNA (as in Fig. 1) are also displayed. qRT-PCR was also used to validate the level of MRB7260 knockdown in the biological replicate experiments (32\%$48 \%)$. (C,E) Comparison of RNA immunoprecipitated with TbRGG2-HTM $(C)$ or MRB3010-PTP $(E)$ compared to a mock IP. RNA was detected using RIP mRNA primers described in $A$ and primers designed to detect a subset of gRNAs using qRT-PCR. RNA levels were standardized against 18S rRNA, and numbers represent the mean and standard deviation of two biological replicates with at least 8 determinations total. $(D, F)$ Comparison of RNA immunoprecipitated with TbRGG2-HTM $(D)$ or MRB3010-PTP $(F)$ grown with or without tet to induce MRB7260 RNAi. Fold change with MRB7260 knockdown represents the RNA levels detected in the RIP from tet-induced cells compared to the RIP from uninduced cells. RNA was detected using RIP mRNA primers described in $A$ and primers designed to detect a subset of gRNAs using qRT-PCR. RNA levels were standardized against $18 \mathrm{~S}$ rRNA and numbers represent the mean and standard deviation of two biological replicates with at least eight determinations total.

association of both mRNA and gRNA with the REMC subcomplex. Next, we wanted to measure the change in the RNA associated with the GRBC subcomplex in the presence or absence of MRB7260. After verification that the RNAs associated with GRBC were enriched compared to a mock IP (Fig. 7E), we then compared the RNA levels in MRB3010-PTP pulldowns replete for or depleted of
MRB7260. GRBC-associated mRNAs were largely unaffected by MRB7260 depletion, with only A6 mRNA slightly decreased (Fig. 7F). In contrast, there was a major decrease in all but one of the gRNAs associated with the GRBC subcomplex in MRB7260-depleted cells (Fig. 7F). Together, these data demonstrate that MRB7260 is required for proper trafficking of both mRNA and gRNA within RESC. 


\section{DISCUSSION}

RESC is a dynamic multiprotein complex, comprised of REMC and GRBC modules, that serves as the platform for the editing reaction and coordinates interactions between mRNA, gRNA, and RECC (Hashimi et al. 2008, 2013; Panigrahi et al. 2008; Weng et al. 2008; Acestor et al. 2009; Ammerman et al. 2010, 2011, 2012, 2013; Hernandez et al. 2010; Aphasizheva et al. 2014; Aphasizheva and Aphasizhev 2016; Read et al. 2016; Simpson et al. 2017). In this study, we address the function of a previously uncharacterized RESC protein, MRB7260, which was originally identified in a subset of RESC pulldowns (Ammerman et al. 2012; Aphasizheva et al. 2014). We show that MRB7260 is mitochondrially localized and essential for optimal parasite growth and RNA editing in both the PF and BF T. brucei life cycle stages. MRB7260 interacts with components of REMC and GRBC that are dissociated from intact REMC or GRBC as they have been previously defined (Ammerman et al. 2011, 2012, 2013; Aphasizheva et al. 2014). MRB7260 depletion studies provide evidence that this protein is essential for the proper protein-protein and protein-RNA interactions within the RESC subcomplexes, specifically promoting gRNA recruitment to GRBC complexes and stabilizing the GRBC-REMC interaction. Highthroughput sequencing analyses reveal that MRB7260 is essential for RNA editing progression through gRNA-directed blocks of RPS12 mRNA, and provide evidence for a role for MRB7260 in gRNA exchange.

Both productive protein-protein interactions and normal RNA trafficking within RESC are facilitated by MRB7260. Specifically, REMC and GRBC subcomplexes fail to efficiently interact with each other in MRB7260-depleted cells, although the individual subcomplexes apparently remain intact. mRNA association with GRBC remains for the most part unchanged under these conditions; however, the levels of gRNA associated with GRBC decrease two- to fourfold. This decrease in gRNA-GRBC interaction is not due to a change in the overall levels of gRNA upon MRB7260 knockdown. Rather, we observe a concomitant accumulation of gRNAs in association with REMC. Therefore, one potential function of the small MRB7260/GAP1-containing complexes is to facilitate gRNA trafficking to GRBC during gRNA exchange. Curiously, the dissociated GRBC and REMC modules in MRB7260-depleted cells both contain GAP1 (presumably within a GAP1/2 heterotetramer [Weng et al. 2008]), and the level of GAP1 interacting with either GRBC or REMC is unchanged upon MRB7260 depletion. That is, the decrease in gRNA associated with GRBC when MRB7260 is depleted is not accompanied by a decrease in GAP1. These findings indicate that there are numerous pools of GAP1/2, and that gRNAs may be transferred between different GAP1/2 tetramers during gRNA exchange. Thus, while previous models have depicted GAP1/2 as a component of GRBC (Ammerman et al. 2012, 2013; Aphasizheva et al.
2014), our data paint a scenario in which, during gRNA exchange, gRNA-loaded GAP1/2 is associated with REMC, and MRB7260 may facilitate the docking of this complex with GRBC.

In this study, we describe previously unreported complexes with rearranged RESC proteins by visualizing MRB7260containing complexes on glycerol gradients. These findings add complexity to our understanding of RESC composition and potential variants. GAP1, in addition to being a GRBC component (Weng et al. 2008; Hashimi et al. 2009; Ammerman et al. 2011, 2013; Aphasizheva et al. 2014), is also a constituent of a smaller RNA-dependent complex containing MRB7260 (Fig. 5). Moreover, GAP1 appears to also interact with REMC independently of GRBC (Fig. 6). The presence of GAP1 in complexes outside of GRBC is reminiscent of REH2C complexes, containing the REH2 helicase and its associated factors ${ }^{\mathrm{H} 2} \mathrm{~F} 1$ and ${ }^{\mathrm{H} 2} \mathrm{~F} 2$ (Madina et al. 2014; Kumar et al. 2016), which exhibit an RNA-dependent interaction with GAP1 in the absence of the core GRBC component, MRB3010 (Kumar et al. 2016). However, REH2C has been proposed to function in both initiation and progression of editing and in recruitment of pre-edited mRNA to GRBC (Kumar et al. 2016). Thus, these two non-GRBC, GAP1-containing complexes apparently perform different functions. This is consistent with the absence of REH2C component, ${ }^{\mathrm{H} 2} \mathrm{~F} 2$, from our MRB7260 immunoprecipitations. It will be of interest in the future to determine if these complexes function sequentially or in parallel pathways to promote editing. Overall, the existence of separate complexes outside of the previously defined REMC and GRBC modules further supports the dynamic nature of RESC.

High-throughput sequencing of mitochondrial mRNAs in MRB7260 replete and depleted cells provided more detailed insights into the steps of RNA editing that are regulated by MRB7260. These studies clearly showed that MRB7260 does not play a role in editing initiation. Pre-edited mRNAs do not accumulate in MRB7260 knockdown cells, and analysis of editing progression using TREAT (Simpson et al. 2016) demonstrates that MRB7260 has no effect on editing through the first gRNA-directed region of RPS12 mRNA. This is in contrast to REMC components TbRGG2, MRB8180, and MRB8170, all of which are necessary for progression through the initial gRNA-directed region of RPS12 (Simpson et al. 2017). Thus, although MRB7260 associates with GAP1 and promotes gRNA association with GRBC, it does not do so for the initiating gRNA, implying the presence of a separate editing initiation machinery that recruits gRNA and does not contain MRB7260. MRB7260 does, however, play an important role in progression of editing though RPS12 regions whose editing is directed by gRNAs $2-7$. Pausing in the absence of MRB7260 occurs in patches that are guided by the more $5^{\prime}$ regions of each gRNA, that is, early in their utilization. This is distinct from TbRGG2, which promotes progression through more $3^{\prime}$ regions of gRNAs (Simpson et al. 2017). We also observed several EPSs in 
MRB7260-depleted cells corresponding to the ends of gRNAs, consistent with a potential role of MRB7260 in gRNA exchange. Remarkably, we identified a class of mRNAs that increased upon MRB7260 depletion with EPSs at end of gRNA-2 that appears to be guided by an unconventional gRNA following this gRNA end. These data suggest that, in the absence of MRB7260, the correct gRNA-3 is often substituted by an incorrect or alternative gRNA. While we cannot rule out the possibility that MRB7260 is involved in the kinetic temporary shufflings and repositioning of gRNA-mRNA complex, the length and position of the alternative sequence are more consistent with the use of an alternative gRNA. Therefore, we hypothesize that MRB7260 may play a role in proofreading gRNA-mRNA interactions during recruitment and early gRNA-mRNA positioning. Expanded TREAT analysis of numerous mRNAs in the presence or absence of MRB7260 will reveal whether this is a common function of this protein, and whether unconventional gRNA usage leads to production of alternative open reading frames.

Collectively, we present three lines of evidence suggesting that MRB7260 functions in gRNA trafficking during trypanosome RNA editing. First, MRB7260 forms a distinct complex with the gRNA binding protein, GAP1. Second, MRB7260 knockdown leads to gRNA accumulation with REMC and depletion from GRBC. Finally, high-throughput sequencing reveals increased editing pausing at early sites following gRNA anchors and at some gRNA ends, as well as apparent increased utilization of an alternative gRNA in MRB7260-depleted cells. These data are consistent with a model in which MRB7260 promotes gRNA recruitment to GRBC, and participates in early gRNA-mRNA positioning and gRNA discrimination. Future studies will be aimed at testing this model.

\section{MATERIALS AND METHODS}

\section{T. brucei culture conditions and generation of cell lines}

Procyclic form (PF) T. brucei strain 29-13, which co-express T7 RNA polymerase and Tet repressor, and all other PF strains were grown under standard conditions (Wirtz et al. 1999; Pelletier and Read 2003). Bloodstream form (BF) single-marker cells were cultured in HMI-11 medium supplemented with $10 \%$ (v/v) FBS and grown under conditions described previously (Hirumi and Hirumi 1989). For construction of RNAi and tagged cell lines, see Supplemental Methods. All primers used in this study are listed in Supplemental Table S2.

Several cell lines harboring MRB7260 knockdown were used in this study, and the degree of knockdown varies depending on the cell line and experiment as detailed in the figure legends. Because specific anti-MRB7260 antibodies were not available until this study was nearly complete, we did not evaluate the level of knockdown at the protein level for most experiments. Supplemental Figure S6 provides an estimate of the correlation between a given degree of knockdown at the protein and RNA levels, using western blot and
qRT-PCR analysis of cells grown at different concentrations of doxycycline.

\section{Pulldowns and western blotting}

For detection of protein steady-state levels in the presence or absence of MRB7260, PF MRB7260 RNAi cells were grown with or without $4 \mu \mathrm{g} / \mathrm{mL}$ tetracycline for $3 \mathrm{~d}$. Whole-cell lysates $\left(5 \times 10^{6}\right.$ cells) were electrophoresed on $10 \%(\mathrm{w} / \mathrm{v})$ SDS-PAGE gels, transferred to nitrocellulose membrane, and probed with the polyclonal antibodies targeting the following proteins: MRB3010 (Ammerman et al. 2012), GAP1 (Ammerman et al. 2011), MRB11870 (Ammerman et al. 2012), MRB10130 (Ammerman et al. 2011), TbRGG2 (Fisk et al. 2008), MRB8180 (Simpson et al. 2017), MRB8170 (Kafková et al. 2012), KREPA6 (Tarun et al. 2008), p22 (Hayman et al. 2001), and ${ }^{\mathrm{H} 2} \mathrm{~F} 2$ (Kumar et al. 2016). Polyclonal antibodies were generated against recombinant MBP-MRB7260-His (Bethyl Laboratories). MRB7260 antibodies were then affinity purified from the rabbit sera. See Supplemental Methods for description of pulldowns.

\section{Glycerol gradients}

Immunoaffinity purification of MRB7260 was carried out as described in the Supplemental Methods with lysates that were either nuclease-inhibited or nuclease-treated. TEV elutions for the RNA+ and RNA- samples were then loaded on $11-\mathrm{mL} 10 \%-30 \%(\mathrm{v} / \mathrm{v})$ glycerol gradients and centrifuged at $38,000 \mathrm{rpm}$ in a Beckman SW41 rotor for $12 \mathrm{~h}$ at $4^{\circ} \mathrm{C}$. Individual $0.5-\mathrm{mL}$ fractions were collected from the top and analyzed using western blot with antibodies described above. This was also performed using T. brucei 29-13 cell lysate.

\section{Immunofluorescence}

To visualize MRB7260 in vivo, PF T. brucei containing the MRB7260-MHT construct and control parental cell line 29-13 were treated with $250 \mathrm{nM}$ MitoTracker Red CMXRos (Invitrogen) for $15 \mathrm{~min}$ to label mitochondria. Cells were harvested by centrifugation and resuspended in phosphate-buffered saline containing $0.1 \%(\mathrm{w} / \mathrm{v})$ glucose $(\mathrm{PBS}-\mathrm{G})$ at $2.5 \times 10^{7}$ cells $/ \mathrm{mL}$. Cells were fixed with $4 \%(\mathrm{v} / \mathrm{v})$ formaldehyde in PBS for $30 \mathrm{~min}$ followed by permeabilization with $0.5 \%(\mathrm{v} / \mathrm{v}) \mathrm{NP}-40$. Cells were blocked using $0.3 \%$ $(\mathrm{w} / \mathrm{v})$ BSA for $30 \mathrm{~min}$ and then incubated with mouse monoclonal anti-Myc antibody [c-Myc(9E10), Santa Cruz] at a dilution of 1:50 in PBS with $0.1 \%(\mathrm{w} / \mathrm{v})$ BSA for $1 \mathrm{~h}$. Alexa Fluor 488 goat antimouse antibody (ThermoFisher) was diluted 1:200 in PBS-BSA and incubated with cells for $30 \mathrm{~min}$. ProLong Gold antifade reagent with $4^{\prime}, 6^{\prime}$-diamidino-2-phenylindole (DAPI) (Invitrogen) was used to mount the cells. Images were taken with a Zeiss Axio Imager.M2 microscope using Volocity software.

\section{High-throughput sequencing and bioinformatic analysis}

PF T. brucei MRB7260 RNAi cells were grown in the presence or absence of $4 \mu \mathrm{g} / \mathrm{mL}$ tetracycline for $3 \mathrm{~d}$ followed by isolation of RNA using TRIzol per manufacturer's instructions. Two biological replicate experiments were performed and qRT-PCR was used to 
validate the level of MRB7260 knockdown (26\%-40\% remaining). cDNA was generated from DNase-treated RNA using gene specific primers (Supplemental Table S2; Simpson et al. 2016). These cDNA samples were PCR amplified within the linear range of PCR to maintain the relative abundance of unique fragments. Amplicons were then sequenced using paired-end Illumina MiSeq and paired as described previously (Simpson et al. 2016). The number of total (decollapsed) and unique (collapsed) reads for each sample are listed in Supplemental Table S3. To normalize the number of reads in each sample, the total (decollapsed) number of fragments that have no non-T mismatches (standard alignments in Supplemental Table S3) are normalized to 100,000 reads. This normalization scales each sample so that their relative abundance can be compared via their normalized counts (Simpson et al. 2016). Two biological replicates of the MRB7260 RNAi were used and compared to 10 uninduced samples, two from this study and 8 from a previous study (Simpson et al. 2017).

TREAT (Trypanosome RNA Editing Alignment Tool) was used as a multiple sequence alignment and visualization tool (Simpson et al. 2016). TREAT consists of a command-line alignment tool along with a built-in web server providing a web-based interface for searching, viewing and analyzing the alignment results. TREAT is written in Go and freely available under the GPLv3 license at http://github.com/ubccr/treat. TREAT v0.03 (Simpson et al. 2017) was used in this study. Analysis of pre-edited transcripts and determination of EPSs was performed as described previously (Simpson et al. 2017). Code for all R analysis used in this paper can be found through the TREAT github repository (ubccr/treat). The sequencing data used in this study has been deposited in Sequence Read Archive, accession number SRP109103 for the MRB7260 samples (two uninduced and two induced) and accession number SRP097727 for the additional 8 uninduced samples from a previous study (Simpson et al. 2017). See Supplemental Methods for explanation of bioinformatic analyses.

\section{RNA immunoprecipitation}

PF T. brucei harboring the MRB7260 RNAi construct along with either the MRB3010-PTP or TbRGG2-HTM construct were grown with or without $4 \mu \mathrm{g} / \mathrm{mL}$ tetracycline for $3 \mathrm{~d}$, and mitochondria were enriched as described previously (Hashimi et al. 2008). Enriched mitochondria were lysed in N150 buffer (50 mM Tris [pH 8], 150 $\mathrm{mM} \mathrm{NaCl}, 0.1 \%[\mathrm{v} / \mathrm{v}] \mathrm{NP}-40$ and $5 \mathrm{mM} \beta-\mathrm{ME})$ with $1 \%(\mathrm{v} / \mathrm{v})$ Triton X-100 in the presence of Complete protease inhibitor cocktail (Roche), RNase inhibitor (Applied Biosystems), and DNase $1(1 \mu \mathrm{g} / \mathrm{mL})$. Lysates were precleared using Superdex 200 (Amersham) and then incubated with either anti-C-myc agarose (ICL) for TbRGG2-HTM or IgG Sepharose 6 Fast Flow beads (GE Healthcare) for MRB3010-PTP. For the mock immunoprecipations, anti-HA antibodies were coupled to protein-G Sepharose (GE Healthcare) (TbRGG2 RIP) or Superdex 200 (Amersham) (MRB3010 RIP) were used. Beads were washed with N150 in the presence of RNase inhibitor and treated with DNase $1(100 \mu \mathrm{g} / \mathrm{mL})$ for $30 \mathrm{~min}$ at $37^{\circ} \mathrm{C}$. Prior to treatment with proteinase, $2 \%$ of beads were subjected to western blot using antibodies against the myc tag for theTbRGG2-HTM RIPs or Protein-C antibodies for the MRB3010-PTP RIPs to normalize the amount of protein immunoprecipitated. RNA was then extracted from the bound protein using proteinase $\mathrm{K}$ treatment followed by phenol/chloroform extraction.
RNA was DNase-treated (Ambion DNA-free DNase Kit) and converted to cDNA with gene specific primers (Supplemental Table S2) using iScript Select cDNA Synthesis Kit (Bio-Rad). Prior to addition to qRT-PCR, cDNA was preamplified using SsoAdvanced PreAmp Supermix. qRT-PCR was then performed as described above using $18 \mathrm{~S}$ rRNA as a normalization gene. All primers used for RIP qRT-PCR are listed in Supplemental Table S2. All end products described here were gel-isolated, cloned and confirmed by sequencing. See Supplemental Methods for RIP analysis.

\section{DATA DEPOSITION}

Code for all $\mathrm{R}$ analysis used in this paper can be found through the TREAT github repository (https://github.com/ubccr/treat). The sequencing data used in this study has been deposited in Sequence Read Archive, accession number SRP109103 for the MRB7260 samples (two uninduced and two induced) and accession number SRP097727 for the additional eight uninduced samples from a previous study (Simpson et al. 2017).

\section{SUPPLEMENTAL MATERIAL}

Supplemental material is available for this article.

\section{ACKNOWLEDGMENTS}

We thank the University at Buffalo Genomics and Bioinformatics Core, especially Sujith Valiyaparambil and Jonathan Bard. We also thank Sarah Metcalfe for technical assistance, Andrew Bruno for assistance with TREAT analysis, and Vandana Iyer for assistance with circular dichroism. This work was supported by the National Institutes of Health (NIH) (AI061580 to L.K.R., AI125982-01 to Y.S. and R.C., DE024523-01 to Y.S.); SUNY Research Foundation (to Y.S. and R.C.). The circular dichroism instrument was provided by grant S10RR027232 from the NIH/National Center for Research Resources (NCRR).

Received December 1, 2017; accepted January 8, 2018.

\section{REFERENCES}

Acestor N, Panigrahi AK, Carnes J, Ziková A, Stuart KD. 2009. The MRB1 complex functions in kinetoplastid RNA processing. RNA 15: $277-286$.

Ammerman ML, Presnyak V, Fisk JC, Foda BM, Read LK. 2010. TbRGG2 facilitates kinetoplastid RNA editing initiation and progression past intrinsic pause sites. RNA 16: 2239-2251.

Ammerman ML, Hashimi H, Novotná L, Cicová Z, McEvoy SM, Lukes J, Read LK. 2011. MRB3010 is a core component of the MRB1 complex that facilitates an early step of the kinetoplastid RNA editing process. RNA 17: 865-877.

Ammerman ML, Downey KM, Hashimi H, Fisk JC, Tomasello DL, Faktorová D, Kafková L, King T, Lukes J, Read LK. 2012. Architecture of the trypanosome RNA editing accessory complex, MRB1. Nucleic Acids Res 40: 5637-5650.

Ammerman ML, Tomasello DL, Faktorová D, Kafková L, Hashimi H, Lukeš J, Read LK. 2013. A core MRB1 complex component is indispensable for RNA editing in insect and human infective stages of Trypanosoma brucei. PLoS One 8: e78015.

Aphasizheva I, Aphasizhev R. 2016. U-insertion/deletion mRNA-editing holoenzyme: definition in sight. Trends Parasitol 32: 144-156. 
Aphasizheva I, Zhang L, Wang X, Kaake RM, Huang L, Monti S, Aphasizhev R. 2014. RNA binding and core complexes constitute the U-insertion/deletion editosome. Mol Cell Biol 34: 4329-4342.

Blum B, Simpson L. 1990. Guide RNAs in kinetoplastid mitochondria have a nonencoded $3^{\prime}$ oligo $(\mathrm{U})$ tail involved in recognition of the preedited region. Cell 62: 391-397.

Carnes J, Trotter JR, Ernst NL, Steinberg A, Stuart K. 2005. An essential RNase III insertion editing endonuclease in Trypanosoma brucei. Proc Natl Acad Sci 102: 16614-16619.

Carnes J, Trotter JR, Peltan A, Fleck M, Stuart K. 2008. RNA editing in Trypanosoma brucei requires three different editosomes. Mol Cell Biol 28: $122-130$.

Carnes J, Schnaufer A, McDermott SM, Domingo G, Proff R, Steinberg AG, Kurtz I, Stuart K. 2012. Mutational analysis of Trypanosoma brucei editosome proteins KREPB4 and KREPB5 reveals domains critical for function. RNA 18: 1897-1909.

d'Avila-Levy CM, Boucinha C, Kostygov A, Santos HL, Morelli KA, Grybchuk-Ieremenko A, Duval L, Votypka J, Yurchenko V, Grellier P, et al. 2015. Exploring the environmental diversity of kinetoplastid flagellates in the high-throughput DNA sequencing era. Mem Inst Oswaldo Cruz 110: 956-965.

Dixit S, Müller-McNicoll M, David V, Zarnack K, Ule J, Hashimi H, Lukeš J. 2017. Differential binding of mitochondrial transcripts by MRB8170 and MRB4160 regulates distinct editing fates of mitochondrial mRNA in trypanosomes. mBio 8: e02288-16.

Fisk JC, Ammerman ML, Presnyak V, Read LK. 2008. TbRGG2, an essential RNA editing accessory factor in two Trypanosoma brucei life cycle stages. J Biol Chem 283: 23016-23025.

Foda BM, Downey KM, Fisk JC, Read LK. 2012. Multifunctional G-rich and RRM-containing domains of TbRGG2 perform separate yet essential functions in trypanosome RNA editing. Eukaryot Cell 11: $1119-1131$.

Gazestani VH, Nikpour N, Mehta V, Najafabadi HS, Moshiri H, Jardim A, Salavati R. 2016. A protein complex map of Trypanosoma brucei. PLoS Negl Trop Dis 10: e0004533.

Güther ML, Urbaniak MD, Tavendale A, Prescott A, Ferguson MA. 2014. High-confidence glycosome proteome for procyclic form Trypanosoma brucei by epitope-tag organelle enrichment and SILAC proteomics. J Proteome Res 13: 2796-2806.

Hashimi H, Zíková A, Panigrahi AK, Stuart KD, Lukes J. 2008. TbRGG1, an essential protein involved in kinetoplastid RNA metabolism that is associated with a novel multiprotein complex. RNA 14: 970-980.

Hashimi H, Cicová Z, Novotná L, Wen YZ, Lukes J. 2009. Kinetoplastid guide RNA biogenesis is dependent on subunits of the mitochondrial RNA binding complex 1 and mitochondrial RNA polymerase. RNA 15: 588-599.

Hashimi H, Zimmer SL, Ammerman ML, Read LK, Lukeš J. 2013. Dual core processing: MRB1 is an emerging kinetoplast RNA editing complex. Trends Parasitol 29: 91-99.

Hayman ML, Miller MM, Chandler DM, Goulah CC, Read LK. 2001. The trypanosome homolog of human p32 interacts with RBP16 and stimulates its gRNA binding activity. Nucleic Acids Res 29: 5216-5225.

Hernandez A, Madina BR, Ro K, Wohlschlegel JA, Willard B, Kinter MT, Cruz-Reyes J. 2010. REH2 RNA helicase in kinetoplastid mitochondria: ribonucleoprotein complexes and essential motifs for unwinding and guide RNA (gRNA) binding. J Biol Chem 285: $1220-1228$.

Hirumi H, Hirumi K. 1989. Continuous cultivation of Trypanosoma brucei blood stream forms in a medium containing a low concentration of serum protein without feeder cell layers. J Parasitol 75: 985-989.

Huang Z, Faktorová D, Křížová A, Kafková L, Read LK, Lukeš J, Hashimi H. 2015. Integrity of the core mitochondrial RNA-binding complex 1 is vital for trypanosome RNA editing. RNA 21: 2088-2102.

Jensen RE, Englund PT. 2012. Network news: the replication of kinetoplast DNA. Aпnu Rev Microbiol 66: 473-491.
Kafková L, Ammerman ML, Faktorová D, Fisk JC, Zimmer SL, Sobotka R, Read LK, Lukes J, Hashimi H. 2012. Functional characterization of two paralogs that are novel RNA binding proteins influencing mitochondrial transcripts of Trypanosoma brucei. RNA 18: 1846-1861.

Koslowsky DJ, Bhat GJ, Read LK, Stuart K. 1991. Cycles of progressive realignment of gRNA with mRNA in RNA editing. Cell 67: 537-546.

Koslowsky D, Sun Y, Hindenach J, Theisen T, Lucas J. 2014. The insectphase gRNA transcriptome in Trypanosoma brucei. Nucleic Acids Res 42: 1873-1886.

Kumar V, Madina BR, Gulati S, Vashisht AA, Kanyumbu C, Pieters B, Shakir A, Wohlschlegel JA, Read LK, Mooers BH, et al. 2016. REH2C helicase and GRBC subcomplexes may base pair through mRNA and small guide RNA in kinetoplastid editosomes. J Biol Chem 291: 5753-5764.

Li F, Herrera J, Zhou S, Maslov DA, Simpson L. 2011. Trypanosome REH1 is an RNA helicase involved with the $3^{\prime}-5^{\prime}$ polarity of multiple gRNA-guided uridine insertion/deletion RNA editing. Proc Natl Acad Sci 108: 3542-3547.

Lukes J, Hashimi H, Zíková A. 2005. Unexplained complexity of the mitochondrial genome and transcriptome in kinetoplastid flagellates. Curr Genet 48: 277-299.

Madina BR, Kumar V, Metz R, Mooers BH, Bundschuh R, Cruz-Reyes J. 2014. Native mitochondrial RNA-binding complexes in kinetoplastid RNA editing differ in guide RNA composition. RNA 20: $1142-1152$.

Maslov DA, Simpson L. 1992. The polarity of editing within a multiple gRNA-mediated domain is due to formation of anchors for upstream gRNAs by downstream editing. Cell 70: 459-467.

McAdams NM, Ammerman ML, Nanduri J, Lott K, Fisk JC, Read LK. 2015. An arginine-glycine-rich RNA binding protein impacts the abundance of specific mRNAs in the mitochondria of Trypanosoma brucei. Eukaryot Cell 14: 149-157.

Panigrahi AK, Zíková A, Dalley RA, Acestor N, Ogata Y, Anupama A, Myler PJ, Stuart KD. 2008. Mitochondrial complexes in Trypanosoma brucei: a novel complex and a unique oxidoreductase complex. Mol Cell Proteomics 7: 534-545.

Peikert CD, Mani J, Morgenstern M, Käser S, Knapp B, Wenger C, Harsman A, Oeljeklaus S, Schneider A, Warscheid B. 2017. Charting organellar importomes by quantitative mass spectrometry. Nat Commun 8: 15272.

Pelletier M, Read LK. 2003. RBP16 is a multifunctional gene regulatory protein involved in editing and stabilization of specific mitochondrial mRNAs in Trypanosoma brucei. RNA 9: 457-468.

Read LK, Göringer HU, Stuart K. 1994. Assembly of mitochondrial ribonucleoprotein complexes involves specific guide RNA (gRNA)binding proteins and gRNA domains but does not require preedited mRNA. Mol Cell Biol 14: 2629-2639.

Read LK, Lukeš J, Hashimi H. 2016. Trypanosome RNA editing: the complexity of getting $\mathrm{U}$ in and taking $\mathrm{U}$ out. Wiley Interdiscip Rev RNA 7: 33-51.

Rusché LN, Cruz-Reyes J, Piller KJ, Sollner-Webb B. 1997. Purification of a functional enzymatic editing complex from Trypanosoma brucei mitochondria. EMBO J 16: 4069-4081.

Schnaufer A, Panigrahi AK, Panicucci B, Igo RP Jr, Wirtz E, Salavati R, Stuart K. 2001. An RNA ligase essential for RNA editing and survival of the bloodstream form of Trypanosoma brucei. Science 291: 2159-2162.

Schofield CJ, McDonough MA. 2007. Structural and mechanistic studies on the peroxisomal oxygenase phytanoyl-CoA 2-hydroxylase (PhyH). Biochem Soc Trans 35: 870-875.

Simpson L, Aphasizhev R, Gao G, Kang X. 2004. Mitochondrial proteins and complexes in Leishmania and Trypanosoma involved in U-insertion/deletion RNA editing. RNA 10: 159-170.

Simpson RM, Bruno AE, Bard JE, Buck MJ, Read LK. 2016. Highthroughput sequencing of partially edited trypanosome mRNAs reveals barriers to editing progression and evidence for alternative editing. RNA 22: 677-695. 


\section{McAdams et al.}

Simpson RM, Bruno AE, Chen R, Lott K, Tylec BL, Bard JE, Sun Y, Buck MJ, Read LK. 2017. Trypanosome RNA Editing Mediator Complex proteins have distinct functions in gRNA utilization. Nucleic Acids Res 45: 7965-7983.

Stuart KD, Schnaufer A, Ernst NL, Panigrahi AK. 2005. Complex management: RNA editing in trypanosomes. Trends Biochem Sci 30: 97-105.

Sturm NR, Simpson L. 1990. Kinetoplast DNA minicircles encode guide RNAs for editing of cytochrome oxidase subunit III mRNA. Cell 61: 879-884.

Sturm NR, Maslov DA, Blum B, Simpson L. 1992. Generation of unexpected editing patterns in Leishmania tarentolae mitochondrial mRNAs: misediting produced by misguiding. Cell 70: 469-476.
Tarun SZ Jr, Schnaufer A, Ernst NL, Proff R, Deng J, Hol W, Stuart K. 2008. KREPA6 is an RNA-binding protein essential for editosome integrity and survival of Trypanosoma brucei. RNA 14: 347-358.

Weng J, Aphasizheva I, Etheridge RD, Huang L, Wang X, Falick AM, Aphasizhev R. 2008. Guide RNA-binding complex from mitochondria of trypanosomatids. Mol Cell 32: 198-209.

Wierzbicki AS, Lloyd MD, Schofield CJ, Feher MD, Gibberd FB. 2002. Refsum's disease: a peroxisomal disorder affecting phytanic acid $\alpha$ oxidation. J Neurochem 80: 727-735.

Wirtz E, Leal S, Ochatt C, Cross GA. 1999. A tightly regulated inducible expression system for conditional gene knock-outs and dominantnegative genetics in Trypanosoma brucei. Mol Biochem Parasitol 99: 89-101. 

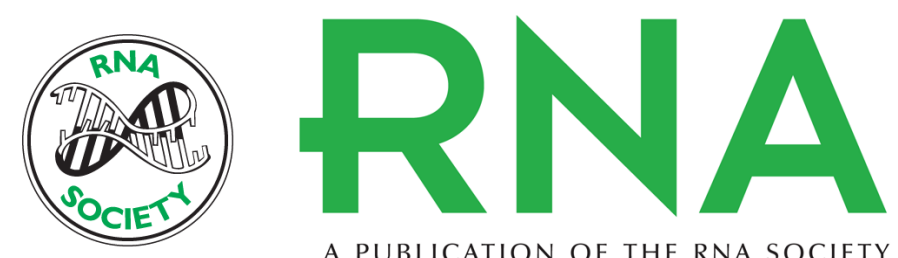

A PUBLICATION OF THE RNA SOCIETY

\section{MRB7260 is essential for productive protein-RNA interactions within the RNA editing substrate binding complex during trypanosome RNA editing}

Natalie M. McAdams, Rachel M. Simpson, Runpu Chen, et al.

RNA 2018 24: 540-556 originally published online January 12, 2018

Access the most recent version at doi:10.1261/rna.065169.117

Supplemental Material

References

Creative Commons License

Email Alerting Service
http://rnajournal.cshlp.org/content/suppl/2018/01/12/rna.065169.117.DC1

This article cites 52 articles, 27 of which can be accessed free at: http://rnajournal.cshlp.org/content/24/4/540.full.html\#ref-list-1

This article is distributed exclusively by the RNA Society for the first 12 months after the full-issue publication date (see http://rnajournal.cshlp.org/site/misc/terms.xhtml). After 12 months, it is available under a Creative Commons License (Attribution-NonCommercial 4.0 International), as described at http://creativecommons.org/licenses/by-nc/4.0/.

Receive free email alerts when new articles cite this article - sign up in the box at the top right corner of the article or click here. 